\title{
Stel Component Analysis: Joint Segmentation, Modeling and Recognition of Objects Classes
}

\author{
Alessandro Perina • Nebojsa Jojic • Marco Cristani • \\ Vittorio Murino
}

Received: 12 November 2010 / Accepted: 11 May 2012 / Published online: 8 June 2012

(C) The Author(s) 2012. This article is published with open access at Springerlink.com

\begin{abstract}
Models that captures the common structure of an object class have appeared few years ago in the literature (Jojic and Caspi in Proceedings of IEEE Computer Society Conference on Computer Vision and Pattern Recognition (CVPR), pp. 212-219, 2004; Winn and Jojic in Proceedings of International Conference on Computer Vision (ICCV), pp. 756-763, 2005); they are often referred as "stel models." Their main characteristic is to segment objects in clear, often semantic, parts as a consequence of the modeling constraint which forces the regions belonging to a single segment to have a tight distribution over local measurements, such as color or texture. This self-similarity within a region in a single image is typical of many meaningful image parts, even when across different images of similar objects, the corresponding parts may not have similar local measurements. Moreover, the segmentation itself is expected to be consistent within a class, although still flexible. These models have been applied mostly to segmentation scenarios.

In this paper, we extent those ideas (1) proposing to capture correlations that exist in structural elements of an image class due to global effects, (2) exploiting the segmentations to capture feature co-occurrences and (3) allowing the use of multiple, eventually sparse, observation of different nature. In this way we obtain richer models more suitable to recognition tasks.
\end{abstract}

\footnotetext{
A. Perina $(\bowtie) \cdot$ N. Jojic

Microsoft Research, Redmond, WA, USA

e-mail: alessandro.perina@gmail.com

\author{
A. Perina $\cdot$ M. Cristani $\cdot$ V. Murino \\ University of Verona, Verona, Italy \\ M. Cristani $\cdot$ V. Murino \\ Italian Institute of Technology, Genova, Italy
}

We accomplish these requirements using a novel approach we dubbed stel component analysis. Experimental results show the flexibility of the model as it can deal successfully with image/video segmentation and object recognition where, in particular, it can be used as an alternative of, or in conjunction with, bag-of-features and related classifiers, where stel inference provides a meaningful spatial partition of features.

Keywords Object class modeling - Statistical learning · Bag of words and beyond $\cdot$ Segmentation $\cdot$ Recognition

\section{Introduction}

Understanding the semantics of an image is one of the most challenging problems in computer vision. A useful object recognition system needs to recognize thousands of objects and learn about new object classes from a relatively small number of examples. It is also essential that learning does not require any human involvement in annotating training images. Image models thus need to encode invariances that would hold across all images, as this reduces the amount of training data.

One of the greater problems in recognizing object classes is that there can be significant changes in appearance from one object instance to another. Real-world imaging conditions and the characteristics of the sensing devices often result in strong fluctuations of pixel intensities, even for similar objects. In addition, the changes in appearance may be due to the variation in material properties among instances of an object. For all these reasons, images are often represented by indices referring to a set of possible local features, more or less invariant to illumination conditions, e.g. SIFT (Lowe 1999), Harris affine regions (Harris and Stephens 
a) Bag of words models

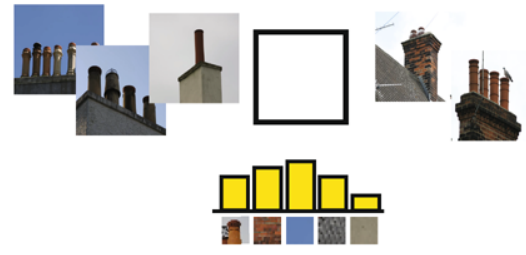

b) Stel component analysis

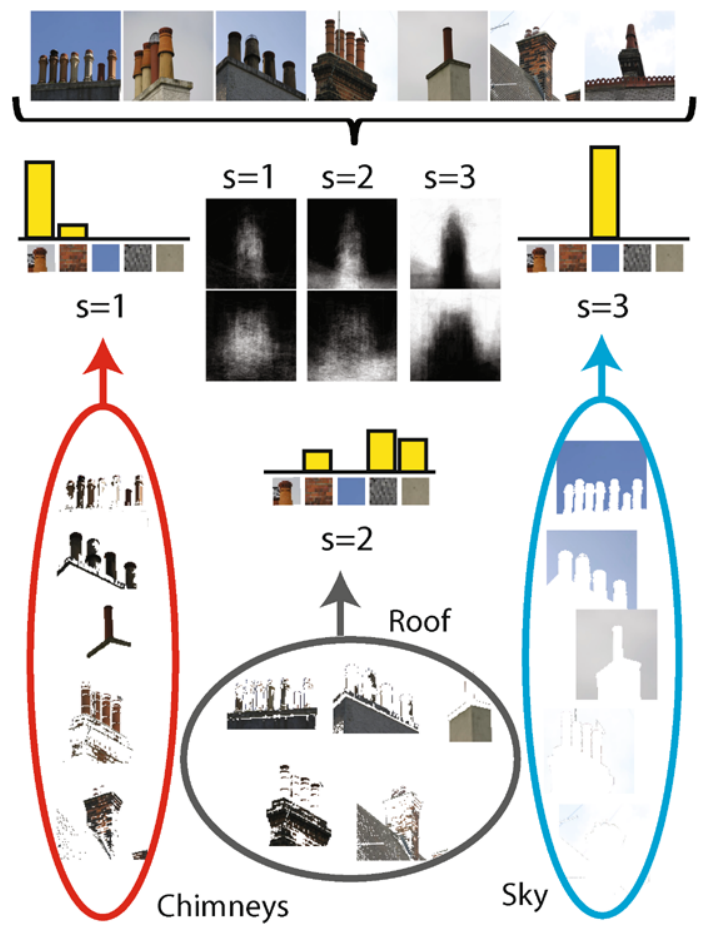

Fig. 1 (a) Bag of words models extract features from the whole images disregarding the spatial information; an histogram of expected feature distribution is then calculated pooling together the features from all the samples. Bag of words is equivalent to stel component analysis with $S=1$. (b) The adaptive-learned feature organization provided by Stel Component Analysis

1988), Maximally Stable Extremal Regions (Matas et al. 2002), and SURF (Bay et al. 2006) to name a few.

In most of the cases, the spatial configuration of the features is not considered: this is the case of bag of words (BoW) models (Fei-Fei and Perona 2005; Dance et al. 2004). In such models, each image class has a distinctive distribution of features, sometimes called "palette". This distribution is typically found in most instances of the class, although the image locations in which these features are found may substantially vary. In any case, the distribution over the feature indices describes an entire image class. Examples of such models are the generative Naive Bayes classifier (Dance et al. 2004), or the generative hierarchical models presented in Fei-Fei and Perona (2005), Hofmann (1999).
An improvement over the bag of words models are the index map models (Jojic and Caspi 2004; Winn and Jojic 2005). Their basic assumption is that within an object class, each image exhibits local intensity patterns that are repeated in nearby image locations in a similar relative geometric layout (Alexe et al. 2010). In other words, although the intra-class appearances of the objects may vary, the object shape is consistent, and the variability of color/texture within a single instance of an object is limited. The literature refers to this assumption as "self-similarity". Beside object class modeling (Jojic and Caspi 2004; Winn and Jojic 2005; Bagon et al. 2010), it has also found its way into object recognition in the form of local (Shechtman and Irani 2007) or global (Deselaers and Ferrari 2010) descriptors.

Under this assumption, another approach to invariance to appearance variation can be used: palette invariance through index map modeling.

We formally define index maps as ordered sets of indices $s_{i} \in 1, \ldots, S$, linked to spatially distinct areas $i \in 1, \ldots, N$ of images where $N$ is the number of such image areas, e.g., pixels (see Fig. 2a). We define an area of an image with the same assigned index $s$ as a structure element.

These indices point to a table of $S$ possible local measurements, referred to as palette. In such model the feature palette is pertinent only to a single instance of a class, while the indexing configuration is assumed to be relevant to the entire class of images.

While the index map models has the advantage over BoW models in terms of capturing the spatial structure of images, it does not deal with (1) the possibility of estimating consistent palettes across images of the class (e.g., in a video sequence, subsequent images do have similar palettes), and (2) possible dependencies among the indices $s_{i}$.

We address both problems by proposing a new model, named Stel Component Analysis (SCA). The model of index variations described above is so enriched by this analysis which, inspired by principle component analysis and other subspace methods, captures correlated variations in discrete indices through a model of blending of several component index maps based on real valued weights $y$. This is visually described in Fig. 2c, where three components $(k=1,2,3)$ are shown and where the blending of them allows for a better agreement of the observed facial image with the model.

In more complex categories, the proposed model benefits from learning a prior over individual image palettes, which is similar to what is achieved in the BoW models, except that these appearance models can now be part-specific. In Fig. 1b, the SCA model is applied to the more complex category of roof images, where the prior over individual image palettes is represented by different histograms over image features in the three different stels. This example illustrates the advantages of the model presented here over both BoW and index models. Where the image class does indeed have 
a) Index Map

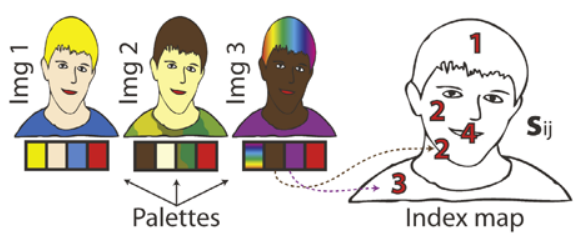

b) Probabilistic Index Map (PIM)

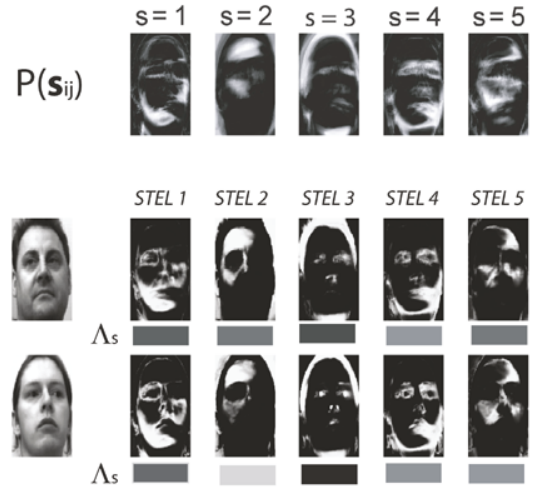

c) Stel Component Analysis (SCA)
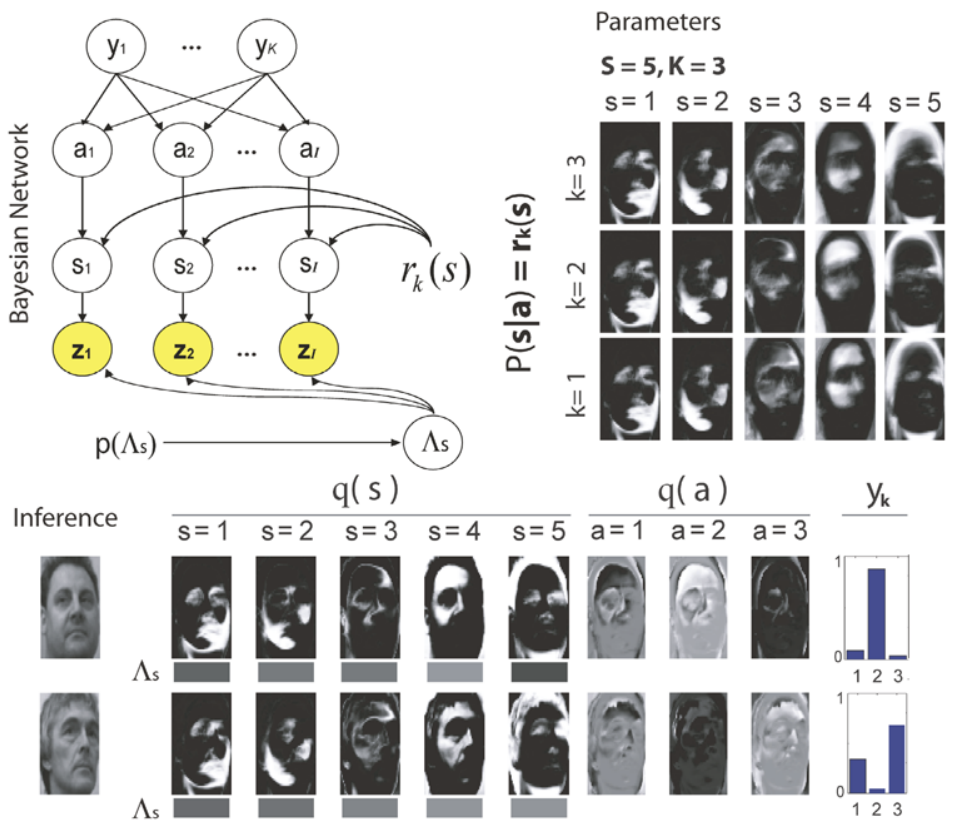
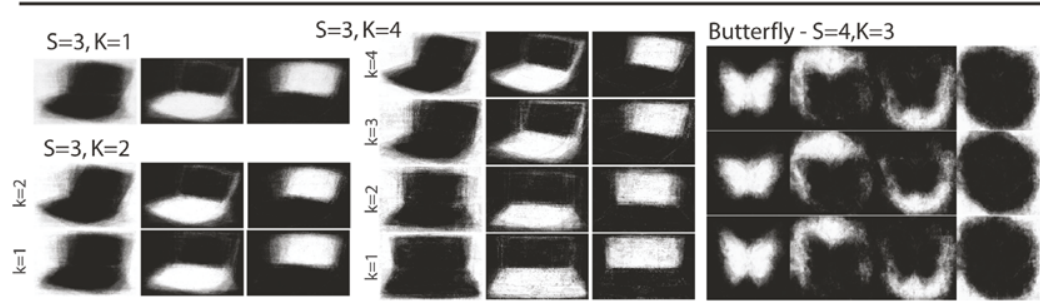

Starfish $-\mathrm{S}=5, \mathrm{~K}=3$

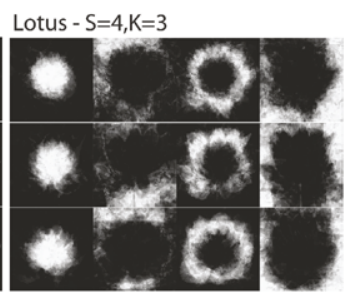

Fig. 2 (a) Illustration of an index map. (b) Probabilistic index map with a palette of size $S=5$-a special case of Stel Component Analysis with $K=1$. (c) Stel component analysis, Bayesian network and inference illustration. SCA with $K=3$ components allows modeling spatially correlated changes in discrete stels. On the bottom, the inferred stels $q(s)$, the image palettes, as well as the component strengths $y_{k}$ for three different faces with varying poses. The strengths $y_{k}$ turn out to be linearly correlated with the pose angle and outperform PCA as pose estimators. Site-specific blending variables $a$ allow nonuniform mixing of components. Their posterior distributions $q(a)$ are also shown

for a better use with discriminative classifiers, (3) we group the stels to perform a foreground extraction or segmentation tasks, (4) we use the stel partitions $q\left(s^{t}\right)$ as feature to capture the global self-similarity of objects.

A previous version of this paper has appeared in Jojic et al. (2009), this paper extends it in several aspects. We extend the experimental section using Caltech 101 instead of an handmade dataset. We compared SCA on image segmentation with (Winn and Jojic 2005; Alexe et al. 2010; Rother et al. 2004) and we evaluated SCA in independent one-vs-all classification tasks.

As technical novelty, we introduce here the derivation for the conjugate priors on the three kind of palette considered and we added a Dirichlet prior on the components $y_{k}$, an overall prior on the stels useful to automatically select the number of stels, and a Markov Random Field prior to prefer that near pixels had the same label. The benefits of these priors will be discussed in the following. Moreover, as further ance object class modeling power of SCA, (2) we the image partitions to group features into meaningful sets 
novelty, we introduce the Stel Kernel, which represents the missing piece of research between the "S-Bags of words" paradigm introduced in Jojic et al. (2009), and our following work (Perina et al. 2010).

The remainder of the paper is organized as follows. Section 2 presents the related work. The details of the architecture of the method and the learning algorithm are presented in Sect. 3. Subsequently, in Sect. 4, experimental results are illustrated. Finally, a critical discussion concludes the paper in Sect. 5.

\section{Related Work}

Previous probabilistic approaches to learning and understanding object classes can be divided based on how they deal with spatial arrangement of local features.

In recent years, bag of words models (Blei et al. 2003), have been successfully used for vision tasks (Fei-Fei and Perona 2005; Bosch et al. 2006; Dance et al. 2004; Willamowski et al. 2004; Jojic et al. 2003; Cristani et al. 2008). These models are particularly attractive due to the computational efficiency and simplicity achieved by ignoring spatial relationships of the image patches or object parts. After extracting the features from images, the features are clustered and a discrete "codewords" is assigned to each feature descriptor. An image is then described by a histogram over the codebook entries.

Topic models (Blei et al. 2003; Hofmann 2001), probably the most famous example among BoW approaches, assign a topic to each codeword based on their co-occurrence in images. A representation that lies between the template and the BoW is the epitome (Jojic et al. 2003, 2010; Ni et al. 2009). It is learned by compiling patches drawn from input images into a condensed image model. The balance between visual resemblance and generalization of image video can be adjusted by the sizes of epitome and patch. Subsequently, Chu et al. (2010), Cheung et al. (2007) present improved epitome models which combined the patch appearance information with some spatial information.

Finally, in Perina and Jojic (2011) the authors present the Counting Grids. The paper shows how much of the variability in vision datasets is better modeled in terms of multidimensional thematic shifts, rather than outright topic mixing proper of topic models (LDA, Blei et al. 2003). Similarly to epitomes, the features are arranged in a window, which is then embedded in some hypothetical bigger scene; certain features are dropped and others added as a consequence of movement in this scene.

In contrast other models explicitly encode spatial information, often at a considerable computational cost. Among these methods, we can identify methods that keep spatial relationships between feature locations and method that spatially organize the features.
In Sivic et al. (2005) is presented a strategy aimed at forming vocabularies from pairs of nearby features called "doublets" or "bigrams". This method, beside taking cooccurrences into account, captures some geometric invariance, but it is too demanding since many doublets have to be estimated. The approach proposed in Leibe et al. (2004) learns a codebook of local appearances that contains information about which local structure may appear on objects of a particular class. It also specifies where a particular codeword may occur on the object. Despite being invariant to rigid transformations, it needs additional supervision through human-supplied object positions and ground truth segmentations.

Generative part-based models (Quattoni et al. 2004; Weber et al. 2000; Savarese and Fei-Fei 2007; Su et al. 2009; Sun et al. 2009; Sudderth et al. 2005), are very nice conceptually and learnable from unsegmented images, but they require a computationally demanding combinatorial hypothesis search. The most famous example is the constellation model (Weber et al. 2000) which attempts to represent an object class by a set of different parts under mutual geometric constraints. The same idea was later used to represent and learn generic 3D object categories (Savarese and FeiFei 2007; Su et al. 2009; Sun et al. 2009).

In Sudderth et al. (2005), it is argued that multi-object recognition systems should be based on models which consider the relationships between different object categories. The approach builds upon the constellation model and it demonstrates that objects classes can be described in terms of shared parts without increasing the size (hence, the complexity) of the representation.

It is also possible to model where the features are present in terms of absolute image locations (Cao and $\mathrm{Li}$ 2007; Marszałek and Schmid 2006; Winn and Jojic 2005; Lazebnik et al. 2006; Vogel and Schiele 2007; Perina et al. 2010; Jojic and Caspi 2004; Jojic et al. 2004). In Cao and Li (2007) propose a generative model, called Spatial-LTM, which assigns topics to an images by incorporating meaningful spatial coherency among the patches. The idea is that pixels should share the same latent topic assignment if they are in a neighboring region with similar appearance.

The well-known Spatial Pyramid Kernel (Lazebnik et al. 2006), extends the BoW paradigm providing a locally orderless representation at several levels of resolution. This is obtained by grouping the features following a hierarchical fixed partition of the images.

Marszałek and Schmid (2006) describe a method to infer the object-background segmentation of test images, starting from labeled training images (i.e., the object extraction mask is known). It classify objects with SVM by weighting the features according to the segmentation.

Index map models (Jojic and Caspi 2004; Winn and Jojic 2005; Jojic et al. 2004) also fall in this category. These 
models aim at capturing the consistent spatial layout of the classes (see Fig. 2, bottom). The probabilistic index map (PIM) approach (Jojic and Caspi 2004; Jojic et al. 2004) relaxes the hard assumption of the index maps that indices that model the same location across the images should be equal, by allowing them instead to following the same distribution. For an example see Fig. 2a (index map).

In this model, each image location $i$ is associated with a distribution over indices $p\left(s_{i}=s\right)$ and each image has its own index map $q\left(s_{i}^{t}\right)^{1}$ governed by $p\left(s_{i}=s\right)$. These indices point to a table of $S$ possible local measurements, referred to as palette.

The PIM model allows for complete freedom of choosing in image-specific color palettes, and thus full paletteinvariance of the indexing model. Figure $2 b$ illustrates a PIM model and it emphasizes the difference between the prior over stels $p(s)$ and the inferred indices for individual images $q\left(s_{i}^{t}\right)$, which depend on both the prior and the self-similarity properties of an individual image.

In Winn and Jojic (2005), the authors build their model, called LOCUS, on the same palette invariance assumption of PIM (Jojic and Caspi 2004), proposing the use of a more expressive color distribution in the entries of the palette, exploiting both appearance and shape (edges), and learning complex priors that capture appearance, edge and color distribution of a class.

Another example of index map model is Perina et al. (2010), where a Latent Dirichlet Allocation model is learned in each segment.

The ideas behind Stel Component Analysis which we present in this paper extend the literature on object class probabilistic models in several respects. First, SCA deals with multiple and sparse features generalizing (Winn and Jojic 2005; Jojic and Caspi 2004) which only relies on dense discrete edge/color and continuous appearance, respectively. We also extended the basic appearance models (palettes) of PIM, presenting much more complex palettes that can capture segments with a multi-modal feature distribution. In contrast to Jojic and Caspi (2004), we cope with the full color invariance, learning the appropriate conjugate priors, that serve to capture interesting co-occurrence of features in the stels. For example, see in Fig. 1b how the third stel for the chimney class models the sky, and many (all) images will present a "bluish" palette in $\Lambda_{s=3}$.

Second, we consider the index maps more broadly than several previous techniques for modeling spatial correlations in index map-like approaches (Shotton et al. 2006; Fei-Fei and Perona 2005; Lazebnik et al. 2006; Jojic and Caspi 2004; Winn and Jojic 2005). Previous models have mostly been limited by three basic characteristics. In one

\footnotetext{
${ }^{1}$ This modeling is due to the variational inference that has been employed, see Sect. 2.
}

class of approaches, a Markov random field is used to define several potentials governing spatial local correlations among few image features (e.g., Shotton et al. 2006). In the second class of methods, a spatial distribution in the image is given for each feature, and this imposes probabilities of seeing a particular index in a particular spot (Fei-Fei and Perona 2005; Lazebnik et al. 2006). In the third one, site-specific distribution over indices, assuming independence in index variation across image locations, are enriched with transformation and deformation models or are used within a mixture model (Jojic and Caspi 2004; Winn and Jojic 2005). Stel component analysis is more flexible than these models, as it captures higher-order statistics than Markov random fields, and can adapt, through its components to a variety of image deformations without parameterizing them ahead of time, as in Jojic and Caspi (2004), Winn and Jojic (2005).

\section{Stel Component Analysis}

\subsection{The Generative Model}

To make image models invariant to changes in local measurements, while sensitive to changes in image structure, a measurement $\mathbf{z}_{\mathbf{i}}^{t}$ (e.g. pixel intensity or feature) at location $\mathbf{i}=(i, j)$ in the $t$-th image of a certain class (object category or a video clip, for instance), is considered to depend on a hidden index $s_{\mathbf{i}}^{t} \in\{1, \ldots, S\}$, Fig. $2 \mathrm{~b}$ :

$p\left(\mathbf{z}_{\mathbf{i}}^{t} \mid s_{\mathbf{i}}^{t}=s\right)=p\left(\mathbf{z}_{\mathbf{i}}^{t} \mid \Lambda_{s}^{t}\right)$.

The $s$-th structure element (stel) indicates pixels $i \mid s_{\mathbf{i}}^{t}=s$ which follow a shared distribution over local measurements (palette) with parameters $\Lambda_{s}^{t}$. In the example in Fig. 2c, each palette entry defines a single Gaussian model with its mean and variance over intensity levels, $\Lambda_{s}^{t}=\left\{\mu_{s}^{t}, \phi_{s}^{t}\right\}$, as was previously done in Jojic and Caspi (2004). The inferred means $\mu_{s}^{t}$ of such palette entries for several facial images are shown in the lower part of each stel. Palettes $\Lambda_{s}^{t}$ are considered hidden variables in the model, each defining a limited diversity of local measurements within each image. However, the stels are generated from a single distribution shared among all images of the class $p\left(\left\{s_{\mathbf{i}}\right\}\right)$ :

$p\left(\left\{s_{\mathbf{i}}\right\}\right)=\prod_{i} p\left(s_{\mathbf{i}}\right)$.

To visualize the class stel distribution, in Fig. $2 \mathrm{~b}$ we show the estimated $p\left(s_{\mathbf{i}}=s\right)$ for $s \in\{1,2,3,4,5\}$ as five images where in the $s$-th image each location indicates the probability of that pixel being mapped to index $s$ for the face pose dataset. The image locations that tend to have similar colors within each individual single image (but not across images) are grouped into stels. 
The pixel intensity $z_{\mathbf{i}}$ tends to be uniform within a stel in a single image, and can be inconsistent across different images, as they may be darker or brighter, for instance. However, the stels are relatively consistent over facial images and they represent interesting image structure beyond intensity levels. For example, stel $s=2$ captures parts of forehead and cheek that have similar surface normals, while the eyebrows and the hair are grouped into stel $s=3$, despite the variability in hair color across images.

The PIM model assumes independence of distributions over indices across different image locations, ignoring the correlations in index variation which often arise even from simple structural variation in the image, such as variation in face proportions, or out of plane head rotation. In models of variation in real valued, rather than discrete, arrays, such correlations are often captured using a subspace model, e.g. PCA, which achieves this through a linear combination of several components. Since we are concerned here with modeling a distribution over discrete indices, we develop a discrete analogue to eigen images, similar in spirit to multinomial PCA (Buntine 2002), non-negative matrix factorization (Lee and Seung 1999), latent Dirichlet analysis models (Blei et al. 2003; Lee and Seung 1999) or the generalization of PCA presented in Collins et al. (2001), but with some important distinctions. Our model is a full probability model of the observed data, meant to capture spatial structure, and thus it is designed for ordered index sets, and it also allows spatially nonuniform mixing of the components.

In stel component analysis the components $r_{k}, k \in$ $\{1, \ldots, K\}$ are of the same form as (2), that is

$r_{k}\left(\left\{s_{\mathbf{i}}\right\}\right)=\prod_{i} r_{k}\left(s_{\mathbf{i}}\right)$.

An example of learned components is shown in Fig. 2c. These components are combined to define the distribution $p\left(\left\{s^{t}\right\}\right)$ using component strengths $y_{k}^{t} \in[0 \ldots 1]$, so that $\sum_{k} y_{k}^{t}=1$. The components strengths are real valued hidden variables for image $t$, rather than component priors in a mixture model as in a mixture of probabilistic index maps. Each image is thus defined by a hidden point in the simplex defined by $\sum_{k} y_{k}^{t}=1$, and this point will rarely fall in a vertex as illustrated in the two inference examples of Fig. 2c, whereas in a mixture model, each image will have a discrete pointer to a single component. In other words, while stel component analysis blends the $K$ components, a mixture of $K$-PIMs would chooses one of the $K$ components.

To achieve this, as well as to allow spatially nonuniform mixing of components based on real valued component strengths, we add a layer of discrete hidden variables $a_{\mathbf{i}} \in\{1, \ldots, K\}$ which act as mixture component indicators, but only locally for their corresponding image locations $\mathbf{i}$ (see the Bayesian network of SCA in Fig. 2c). Hidden component strengths $y_{k}$, shared across the pixels of an image, then act as prior probabilities in these local mixture models:

$$
\begin{aligned}
& p\left(\left\{s_{\mathbf{i}}^{t}\right\} \mid\left\{a_{\mathbf{i}}^{t}\right\}\right)=\prod_{\mathbf{i}} p\left(s_{\mathbf{i}}^{t} \mid a_{\mathbf{i}}^{t}\right) \\
& p\left(s_{\mathbf{i}}^{t} \mid a_{\mathbf{i}}^{t}=k\right)=r_{k}\left(s_{\mathbf{i}}^{t}\right) ; \quad p\left(a_{\mathbf{i}}^{t}=k \mid y_{k}^{t}\right)=y_{k}^{t} .
\end{aligned}
$$

By summing over hidden variables $a$, a desired mixing of components with real valued weights $y_{k}^{t}$ is achieved to form a differently mixed stel distribution for each image. Since different hidden variables $a_{\mathbf{i}}$ can have different values (and thus choose different components $r_{k}$ in different parts of the image), the mixing is spatially nonuniform, and each variable $y_{k}$ influences only the total number of image locations $\mathbf{i}$ that choose $r_{k}\left(s_{\mathbf{i}}\right)$ as the local prior on the index. This allows dramatically more flexible mixing than in PCA models, making object part alignment across images much easier to achieve without global image transformations.

Again, the use of the layer of hidden variables $a_{\mathbf{i}}$ makes the model different from a simple mixture of site-specific models. Index probabilities from different components are blended differently in different parts of the image, which simple mixture models do not allow. This gives the model more flexibility in parsing images, and, as desired, allows for variable mixing of the components for different images to model smooth geometric changes (see Fig. 2c).

The joint distribution over all observed variables $\mathbf{z}=\left\{\mathbf{z}_{\mathbf{i}}^{t}\right\}$, and hidden variables $\mathbf{h}=\left\{\left\{y_{k}^{t}\right\},\left\{a_{\mathbf{i}}^{t}, s_{\mathbf{i}}^{t}\right\},\left\{\Lambda_{s}^{t}\right\}\right\}$ is

$$
\begin{aligned}
p(\mathbf{z}, \mathbf{h})= & \prod_{t}\left(p\left(\left\{y_{k}^{t}\right\}_{k=1}^{K}\right) p\left(\left\{\Lambda_{s}^{t}\right\}\right) \prod_{i} p\left(z_{\mathbf{i}}^{t} \mid s_{\mathbf{i}}^{t},\left\{\Lambda_{s}^{t}\right\}\right)\right. \\
& \left.\times \prod_{k}\left(y_{k}^{t} r_{k}\left(s_{\mathbf{i}}^{t}\right)\right)^{\left.\left[a_{\mathbf{i}}^{t}=k\right]\right)}\right)
\end{aligned}
$$

where $[\cdot]$ is the indicator function. The prior on $y_{k}$ can be kept flat (as in our experiments), or learned in a Dirichlet form.

\subsection{Inference}

Following the variational inference recipe, we (i) introduce a tunable distribution $q(\mathbf{h})$ over the hidden variables/parameters, (ii) define as a bound on the log likelihood $-\log p(\mathbf{z})$, the negative free energy $-F=\sum_{\mathbf{h}} q(\mathbf{h}) \times$ $\log \frac{q(\mathbf{h})}{p(\mathbf{z}, \mathbf{h})}$, and (iii) pursue the strategy of minimizing this free energy iteratively. We used the simplest of the algorithms from this family, where the approximate posterior distribution $q(\mathbf{h})$ is fully factorized

$q(\mathbf{h})=\prod_{k} q\left(r_{k}\right) \prod_{i, t} q\left(a_{i}^{t}\right) q\left(s_{i}^{t}\right) \prod_{t} q\left(y_{k}^{t}\right) q\left(\left\{\Lambda_{s}^{t}\right\}\right)$

with $q\left(r_{k}\right), q\left(y_{k}^{t}\right)$ and $q\left(\Lambda_{s}^{t}\right)$ being Dirac functions centered at the optimal values (or vectors) $\hat{r}_{k}, \hat{y}_{t}^{k},\left\{\hat{\Lambda}_{s}^{t}\right\}$. As a result, 
a)

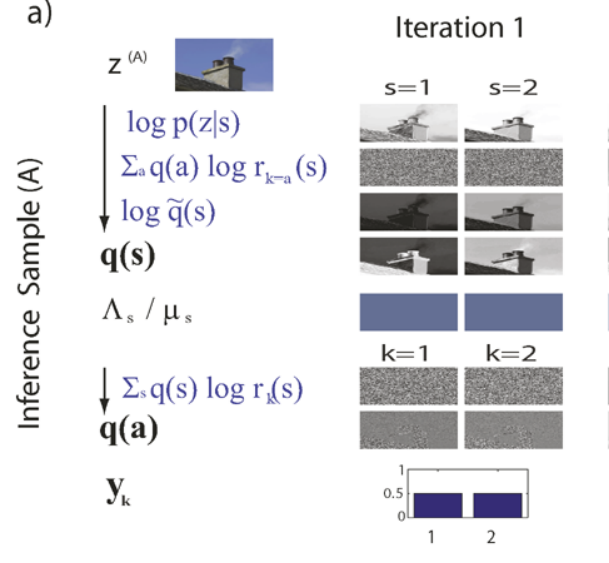

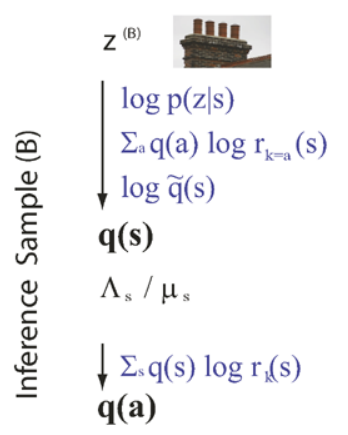

$\mathbf{y}_{\mathrm{k}}$

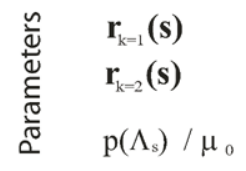
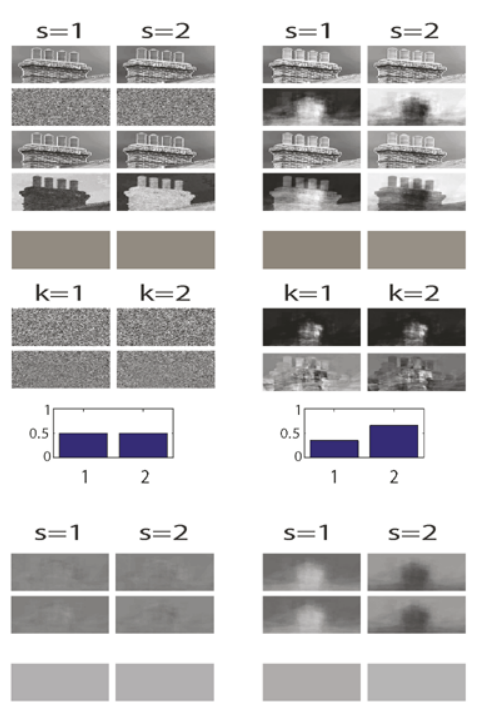
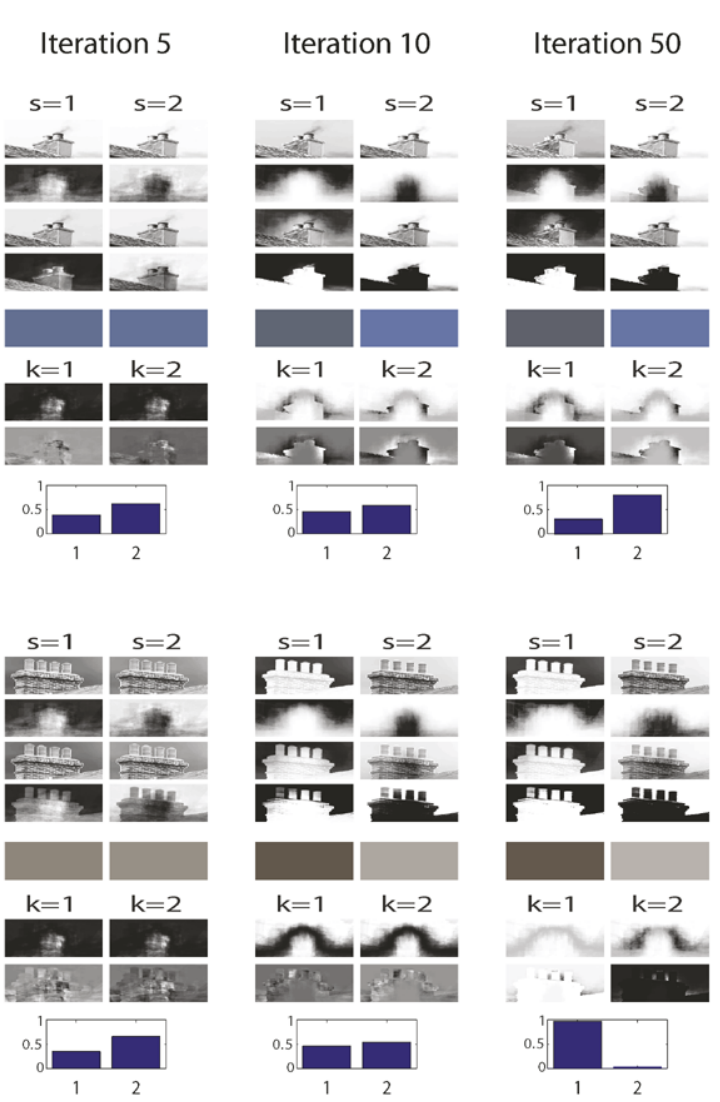

b)
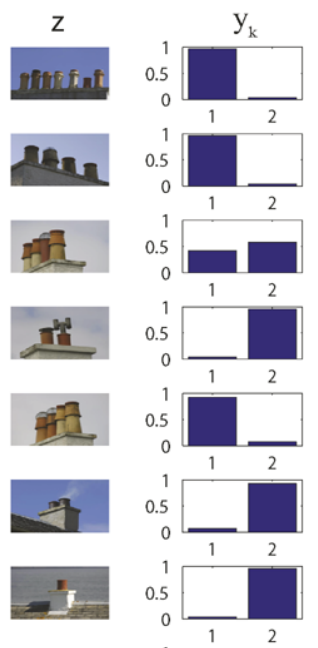

5
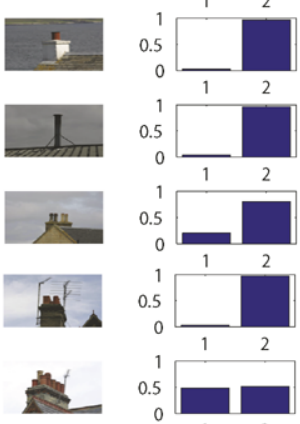

1
0.5
0
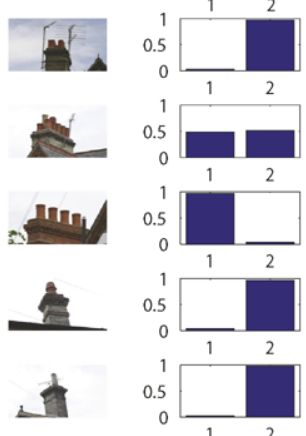

Fig. 3 (a) Illustration of the learning process across the iterations; we show the posterior distributions $\left(q(s), q(a), y_{k}\right.$ and $\Lambda_{s}$, we used black text), the "addends" that compose them (blue text) and the model pa-

rameters. (b) Several images $(z)$ and their components strength $\left(y_{k}\right)$. The final parameters are boxed in the right-bottom most corner of part (a)

$$
\begin{aligned}
\hat{\Lambda}_{s}^{t}= & \arg \max \log p\left(\left\{\hat{\Lambda}_{s}^{t}\right\}\right) \\
& +\sum_{i, s} q\left(s_{i}^{t}=s\right) \log p\left(z_{i}^{t} \mid s_{i}^{t}=s,\left\{\hat{\Lambda}_{s}^{t}\right\}\right) .
\end{aligned}
$$

$$
\begin{aligned}
F= & \sum_{t} p\left(\left\{\hat{\Lambda}_{s}^{t}\right\}\right)+\sum_{t, i, s} q\left(s_{i}^{t}=s\right) \log p\left(z_{\mathbf{i}}^{t} \mid s_{\mathbf{i}}^{t},\left\{\hat{\Lambda}_{s}^{t}\right\}\right) \\
& +\sum_{t, i, k} q\left(a_{i}^{t}=a\right) \log \hat{y}_{a}^{t} \\
& +\sum_{t, i, a, s} q\left(a_{i}^{t}=a\right) q\left(s_{i}^{t}=s\right) \log \hat{r}_{k}\left(s_{i}^{t}=a\right),
\end{aligned}
$$

which is reduced by the following steps, also illustrated in Fig. 3a:

1. The palettes for different stels in a single image $t$ are assigned so as to balance the need to agree with the prior $p(\Lambda)$ with the statistics of the local measurements within a (probabilistic) stel in the image:

More details will be given below, when we will introduce complex palettes.

2. Stel segmentation of image $t$ is based on the similarity of observed local measurements to what is expected in a particular stel $s$ according to the estimated palette $\hat{\Lambda}_{s}^{t}$ in this particular image, as well as the expected stel assignment based on mixed components $r_{k}(s)$. These mixed components are mixed differently in different parts of the image, and the mixing is defined by $q(a)$ :

$q\left(s_{i}^{t}=s\right) \propto p\left(z_{i}^{t} \mid s_{i}^{t},\left\{\hat{\Lambda}_{s}^{t}\right\}\right) e^{\sum_{a} q\left(a_{i}^{t}=a\right) \hat{r}_{a}\left(s_{i}^{t}=s\right)}$.

To avoid numerical underflow, it is useful compute $\log \tilde{q}(s)$ in the log-domain and then normalize. This re- 
duces to

$$
\begin{aligned}
\log \tilde{q}\left(s_{i}^{t}=s\right)= & \log p\left(z_{i}^{t} \mid s_{i}^{t},\left\{\hat{\Lambda}_{s}^{t}\right\}\right) \\
& +\sum_{a} q\left(a_{i}^{t}=a\right) \hat{r}_{a}\left(s_{i}^{t}=s\right) .
\end{aligned}
$$

3. The spatially nonuniform component mixing, defined by $q(a)$, is updated so as to balance the agreement with the overall strength $y_{a}^{t}$ of the component $a$ in the particular image $t$, with the agreement of the stel assignment with the stel component $r_{a}$ :

$$
q\left(a_{i}^{t}=a\right) \propto y_{a}^{t} e^{\sum_{s} q\left(s_{i}^{t}=s\right) \log r_{a}\left(s_{i}^{t}=s\right)} .
$$

As for the previous update rule, to keep numerical precision, one should work in the log-domain.

4. The stel component strengths $y_{a}$ are assigned proportionally to their use in the image:

$y_{a}^{t} \propto \sum_{i} q\left(a_{i}^{t}=a\right)$.

5. The stel components $r_{a}$ are updated to reflect the assignment statistics over all images:

$$
r_{a}(s) \propto \sum_{t} q\left(a_{i}^{t}=a\right) q\left(s_{i}^{t}=s\right) .
$$

Iterating these updates results in learning the model parameters, as well as inferring the hidden variables, e.g., the consistent parsing of images from a class into its stels. With consistent we mean that the same stel represent the same semantic concept in all the images; for example stel 3 in Fig. 2b represent the hair, stel 3 in Fig. 1 always represent the sky, etc.

Figure $3 \mathrm{a}$, also shows the behavior of the learning algorithm; palettes and segmentations $(q(s))$ are immediately learnt, and they converge in few iterations (see posterior at iteration 10 in Fig. 3). After that the model separates and learns the $K$ components to better fit with the data; during this time, the segmentations are slightly refined to capture finer details, until convergence which usually occurs in 60100 iterations. Note in fact, how in Fig. 3 the two components are nearly identical after 10 iterations, while they look very different at convergence.

The energy minimization procedure is illustrated with Algorithm 1. To speed-up the inference process, we performed $E_{i n t}=2,3$ internal iterations of the E-step to gain confidence about $q(s)$ and the palettes. If palette priors are learnt, re-initializing every 10-20 iterations the palettes, possibly using the prior as initial guess, also speeds-up the learning procedure.

To avoid local minima, the palette variances/probabilities and the prior and posterior probabilities on the random variables $s, a$ and $y_{k}$ were not allowed to drop below a small constant $\left(10^{-2} / 10^{-100}\right)$.

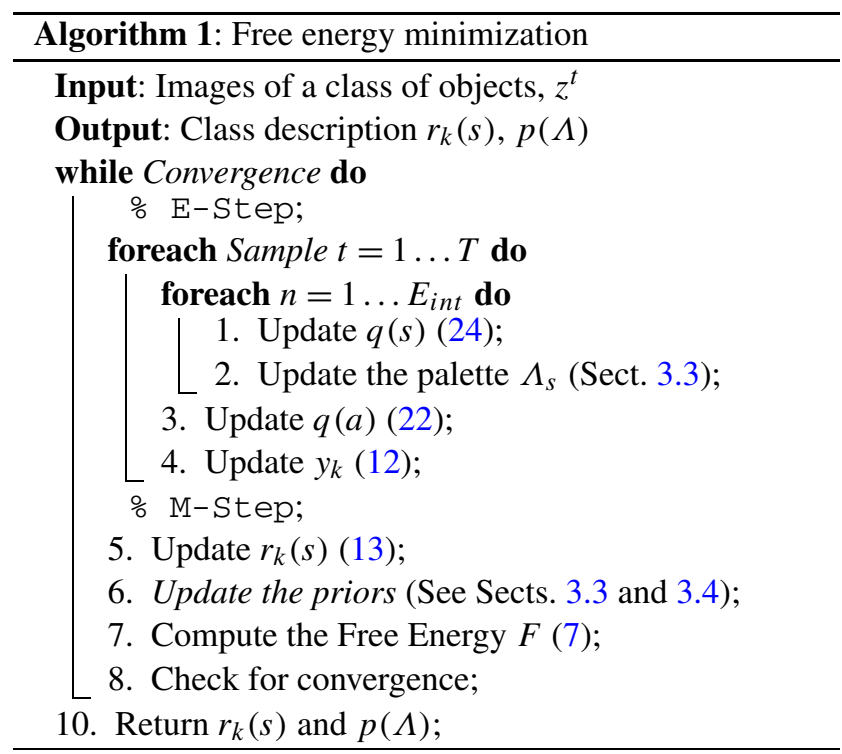

3.3 Local Measurements $z^{t}$, Palette Models $p\left(z^{t} \mid \Lambda_{s}^{t}\right)$ and Palette Priors $p\left(\Lambda_{s}\right)$

The local measurements $z_{i}$ may vary depending on the application, and can be scalar or multidimensional, discrete or real valued. To obtain the face model in Fig. 2, as in (Jojic and Caspi 2004), we assumed that (i) the local measurements are simply the real valued image intensities, (ii) the palette model $\Lambda_{s}=\left(\mu_{s}, \phi_{s}\right)$ is Gaussian, $p\left(z_{i}^{t} \mid s_{i}^{t}=\right.$ $\left.s,\left\{\hat{\Lambda}_{s}^{t}\right\}\right)=\mathcal{N}\left(z_{i}^{t} ; \mu_{s}, \phi_{s}\right)$, (iii) the prior on the palette $\Lambda_{s}$ is kept flat. The palette update is therefore based on sufficient statistics over intensities within stels in individual images, that is

$\mu_{s}^{t} \propto \sum_{i} q\left(s_{i}=s\right) \cdot z_{i}^{t}$

$\phi_{s}^{t} \propto \sum_{i} q\left(s_{i}=s\right) \cdot\left(z_{i}^{t}-\mu_{s}^{t}\right) \cdot\left(z_{i}^{t}-\mu_{s}^{t}\right)^{T}$.

Alternative local measurements include color, disparity, flow, SIFT or some other local features (Mikolajczyk and Schmid 2004). As more expressive palette models, we introduce here the histogram representation for discrete local measurements, and the mixture of Gaussians for the real valued measurements.

For the case of discrete measurements, we define the palette as a histogram over $C$ possible observations $\left\{\zeta_{j}\right\}, j \in$ $\{1, \ldots, C\}$. The observation distribution is multinomial with parameters $u_{j}=p\left(z=\zeta_{j}\right)$, and the palettes $\Lambda_{s}=\left\{u_{s, j}\right\}$ are defined by using these probabilities. With a flat prior on $\Lambda$, (14) reduces to

$u_{s, j}^{t} \propto \sum_{i} q\left(s_{i}=s\right)\left[z_{i}^{t}=\zeta_{j}\right]$.

When measurements consist of different modalities, which are generally uncorrelated at the local level when viewed 
without regard for the high level context, they are combined by setting

$$
p\left(z_{i} \mid s\right)=\prod_{m} p\left(z_{m, i} \mid \Lambda_{m, s}\right)=\prod_{m} \prod_{j} u_{m, s, j}^{\left[z_{m, i}=\zeta_{m, j}\right]}
$$

where $m$ denotes different modality, for example available pixel label and discrete texture features associated with each pixel.

To avoid complete palette invariance, we also add a Dirichlet prior on the histogram palette models:

$p(\Lambda)=p\left(\left\{u_{j}\right\}\right)=\frac{1}{Z\left(\left\{\alpha_{j}\right\}\right)} \prod_{j} u_{j}^{\alpha_{j}-1}$,

which is estimated from the data iteratively together with other updates. The effect of this prior on the palette updates in (15) for different modalities $m$ is $u_{m, s, j}^{t} \propto \alpha_{m, s, j}-1+$ $\sum_{i} q\left(s_{i}=s\right)\left[z_{m, i}^{t}=\zeta_{m, j}\right]$, and the appropriate update on palette priors $\alpha_{j}$ can be shown to be:

$$
\begin{gathered}
\left\{\hat{\alpha}_{s, m, j}\right\}=\arg \max \sum_{t}\left(\alpha_{s, m, j}-1\right) \log u_{m, s, j}, \\
\text { subject to } \sum_{u} \frac{1}{Z\left(\left\{\alpha_{j}\right\}\right)} \prod_{j} u_{j}^{\alpha_{j}-1}=1 .
\end{gathered}
$$

The addition of the (learnable) prior over palette entry allows the model to discover and exploit consistency of local measurements across instances of a class, if there is any.

In case of real valued measurements of arbitrary dimensionality, the palette entry is defined by a mixture of $C$ Gaussians, and the appropriate palette priors are added similarly as in the case of discrete measurements. Being a mixture, each palette entry $\Lambda_{s, c}=\left\{\pi_{s, c}, \mu_{s, c}, \phi_{s, c}\right\}$, and thus the generative model, has a hidden variable $c_{\mathbf{i}}^{t}$ pointing to one of the $C$ Gaussians, which is linked to the observation in the Bayesian network.

With a flat prior on $\Lambda,(8)$ reduces to

$$
\begin{aligned}
\mu_{s, c}^{t} \propto & \sum_{i} q\left(s_{i}^{t}=s, c_{i}^{t}=c\right) \cdot z_{i}^{t} \\
\phi_{s, c}^{t} \propto & \sum_{i} q\left(s_{i}^{t}=s, c_{i}^{t}=c\right) \cdot\left(z_{i}^{t}-\mu_{s, c}^{t}\right) \\
& \cdot\left(z_{i}^{t}-\mu_{s, c . j}^{t}\right)^{T} \\
\pi_{s, c}^{t} & \propto \sum_{i} \sum_{s} q\left(s_{i}^{t}=s, c_{i}^{t}=c\right)
\end{aligned}
$$

where $q\left(s_{i}^{t}=s, c_{i}^{t}=c\right)$ can be further factorized like in (6), and $\pi_{s, c}^{t}$ are the mixing proportions.

The appropriate priors in this case are Gaussians with parameters $\mu_{0_{s, c}}, \psi_{0_{s, c}}$ over the means, scaled inverse Gammas of parameters $\mathbf{a}_{s, c}, \mathbf{b}_{s, c}$ over the variances and Dirichlet distributions $\beta_{s, c}$ over the mixing proportions. The effect of the prior on the palette update rules turns to be

$$
\begin{aligned}
\mu_{s, c}^{t} \propto & \frac{\sum_{i} q\left(s_{i}^{t}=s, c_{i}^{t}=c\right) \cdot z_{i}^{t}}{\phi_{s, c}^{t}}+\frac{\mu_{0_{s, c}}}{\phi_{0_{s, c}}} \\
\phi_{s, c}^{t}= & \frac{\sum_{i} q\left(s_{i}^{t}=s, c_{i}^{t}=c\right) \cdot\left(z_{i}^{t}-\mu_{s, c}^{t}\right) \cdot\left(z_{i}^{t}-\mu_{s, c . j}^{t}\right)^{T}}{\sum_{i} q\left(s_{i}^{t}=s, c_{i}^{t}=c\right)+2 \cdot \mathbf{a}_{s, c}-2} \\
& +\frac{2 \cdot \mathbf{b}_{s, c}}{\sum_{i} q\left(s_{i}^{t}=s, c_{i}^{t}=c\right)+2 \cdot \mathbf{a}_{s, c}-2} \\
\pi_{s, c}^{t} \propto & \beta_{s, c}+\sum_{i} \sum_{s} q\left(s_{i}^{t}=s, c_{i}^{t}=c\right) .
\end{aligned}
$$

Like in the previous cases, each prior is estimated from the data; $\mu_{0_{s, c}}, \psi_{0_{s, c}}$ are equal to the mean and the variance of the palette mean $\mu_{s, c}$ respectively, $\mathbf{a}_{s, c}, \mathbf{b}_{s, c}$ can be estimated fitting a $\Gamma$-function, and the update for $\beta$ is the same as in (18).

An example of this learned prior can be found in Fig. 5, together with the results related to the video segmentation.

When raw local measurements are real valued we can use both discrete and real valued models. The former is achieved by discretizing the measurements by a separate clustering of local measurements to create a codebook. In our experiments, mixture modeling within palette entries was superior to a forced discretization of features outside the full model (as also confirmed by Boiman et al. 2008), but this increased the computational cost. Finally discrete and real valued modalities can be combined, in the same way the multiple discrete modalities are (see for example $\mathrm{Ni}$ et al. 2009).

It is worth mentioning that the use of high dimensional features (e.g. filter bank responses) may cause the observation likelihood to overwhelm the model $r_{k}(s)$. This is due to the fact that so derived features tend to be correlated, and so the model's treatment of them as independent variables leads to over-counting the evidence. The remedy to this is to either use dimensionality reduction (PCA, pLSA or LDA depending on the nature of the observation) or to scale the likelihood terms as is often done in speech research. Actually, in the latter case, it is a standard practice to raise the observation likelihood in HMMs to a power less than 1, before inference is performed on the test sample, as the acoustic signal would otherwise overwhelm the hidden process modeling the language constraints (Deng and O'Shaughnessy 2003).

\subsection{Stel and Components Priors}

The selection of the number of components $K$ and the number of stels $S$ is the first choice to do before learning a SCA's model. Despite this choice is intuitive and not critic, we introduce some prior to help; the idea is to overestimate $S$ and $K$, and let the priors annihilate some of the unused stels/components. 
The number of components $K$ depend on the variability of the viewpoints/scales in the images of the objects. Nevertheless also using 2 components, we are guarantee to do better than Winn and Jojic (2005), Jojic and Caspi (2004). Empirically, to avoid overtrain, at least 10-20 images per component are needed.

When a larger training set is available, we can introduce a Dirichlet prior on $y_{k}$, similar in spirit to LDA (Blei et al. 2003).

$p\left(\left\{y_{k}^{t}\right\}\right)=\frac{1}{Z\left(\left\{\omega_{k}\right\}\right)} \prod_{k}\left(y_{k}^{t}\right)^{\omega_{k}-1}$.

At this point, if SCA has sufficient components to model the data well, it becomes relatively invariant to an increase in $K$ i.e., the additional components should be seldom used (Wallach et al. 2009). The update (12) turns to be

$q\left(a_{i}^{t}=a\right) \propto\left(y_{a}^{t} e^{\sum_{s} q\left(s_{i}^{t}=s\right) \log r_{a}\left(s_{i}^{t}=s\right)}\right)^{\omega_{k}-1}$.

Differently from $K$, the number of stels $S$, also depends on the particular object class. Before discussing the priors, it is important to note that since objects have difference appearance from their surroundings (Alexe et al. 2010; Liu et al. 2007); this is the same assumption upon which relies (Winn and Jojic 2005). Therefore choosing $S=2$ will always segment the image in background and foreground. ${ }^{2}$

Nevertheless one may capture finer details in the object class and want to set $S>2$. This is not always possible; for example pedestrian images can be broken several stels one for each leg, one for each arm, one for the head and so on (Jojic and Caspi 2004), but on the other hand, for less complex classes, like Caltech's barrels, chandeliers or Joshua trees, we can only expect to segment the object from the background.

We can solve this issue learning an overall prior on the stel usage and letting it kill some unused stels.

$\pi_{s} \propto \sum_{t} \sum_{i} q\left(s_{i}^{t}=s\right)$.

The prior looks very similar to $y_{k} \mathrm{~s}$ but it works at class level; it represent how much an object class uses a particular stel. The new update rule for (9) becomes

$q\left(s_{i}^{t}=s\right) \propto \pi_{s} \cdot p\left(z_{i}^{t} \mid s_{i}^{t},\left\{\hat{\Lambda}_{s}^{t}\right\}\right) e^{\sum_{a} q\left(a_{i}^{t}=a\right) \hat{r}_{a}\left(s_{i}^{t}=s\right)}$.

Finally we can also introduce a Markov Random Field (MRF) which enforce smoothness across pixel labels; this has proven to be very useful when the final application is image segmentation (Winn and Jojic 2005; Yang et al. 2010).

${ }^{2}$ Choosing $S=2$ was not possible for Jojic and Caspi (2004) because of its unimodal palette.
3.5 Relationship to Other Models

We can express many other models frequently used in vision and elsewhere as special cases of SCA by assuming an appropriate number of stels $S$, components $K$, and palette entry size $C$. The color histogram model and the bag of words/ features model (Blei et al. 2003; Fei-Fei and Perona 2005; Dance et al. 2004) are achieved with $S=1$. On the other hand, when $S>1$, a single component $r_{k}(s)$ is used, $K=1$, and each palette entry represents a single Gaussian, $C=1$, and the prior over palettes is fixed to flat, our model reduces to a probabilistic index map (PIM) (Jojic and Caspi 2004). Finally, we get the basic ingredient of LOCUS (Winn and Jojic 2005) when we set $S=2$ (foreground/background), and use a large $C$ to represent color histograms in each palette entry.

Both LOCUS and PIM contained transformation variables, which capture correlations due to a given set of simple 2D geometric transformations, while stel component analysis learns (approximately) arbitrary correlations in possible index assignments across an image. The palette choices we discuss here apply to all three models. Finally if we fix the stel partition $q\left(s_{i}^{t}\right)$ to a manual division of the image into square regions, rather than let them be estimated from images themselves, the model becomes similar to Lazebnik et al. (2006).

In contrast to histogram/bag-of-words models, our model parses the images so that the such models are applied only in appropriate parts of different images (thus ignoring the variable background (Marszałek and Schmid 2006), for example). In addition, the likelihood depends on the structure of the image, i.e., the extent to which the parsing of an image into stels is consistent with such parsing of other images in the same category. In this way our model is similar to the PIM model, as it can identify structural similarities among images even in presence of high variations in local measurements across images (but not within a single stel in a single image). But, in addition, stel component analysis allows for a more powerful modeling of these structural characteristics, as well as capturing, if any, feature co-occurrences in the same stel across all images.

\section{Experiments}

The experimental section is divided in three parts. In the first, it is shown how pixels' correlations are captured and how components are blended to better describe an object class. The second part shows how SCA deals with image and video segmentation. In the third part, SCA is used for object classification. We reported classification accuracies on the Caltech 28 dataset (Cao and Li 2007) showing how increasing the components helps the recognition. More important, 
Pose Estimation $-\mathrm{S}=5, \mathrm{~K}=2$

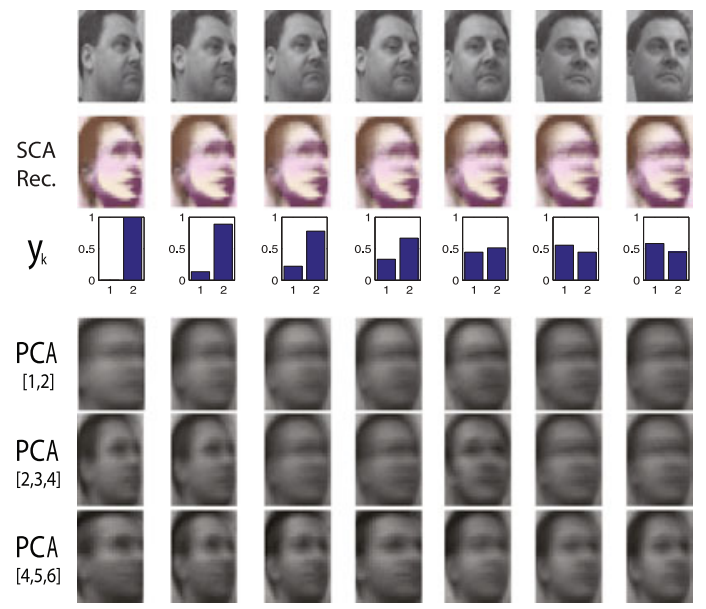

Fig. 4 SCA component strengths $y_{k}, K=2$, for a set of images of faces with varying pose, have a single degree of freedom $\left(y_{1}+y_{2}=1\right)$ and this degree of freedom captures the pose angle well. Below the $y_{k}$ strengths, we show images generated from the model using the $y_{k}$ inferred from the input. The rest of the figure illustrates the PCA reconstruction, which does not manage to separate pose from other causes of variability

on Catlech 101 (Fei-Fei et al. 2007), we used $q\left(s^{t}\right)$ directly as features and we defined the "Stel Kernel" (SK) showing how SCA partitions $q\left(s^{t}\right)$ can be used in to organize other classifiers.

Before each test we show a table to indicate which modality/ies we used, their domain (discrete/continuous), and if we learned $(\checkmark)$ or not $(\times)$, the overall prior on the stels $\pi_{s}$, the Markov Random Field prior on $s$ and the palette prior $P\left(\Lambda_{s}\right)$.

In all the tests, we assume the presence of one object in the image and a bounding-box annotation which can be coarse (i.e. not precisely cropped).

\subsection{Evaluation of the Components}

\subsubsection{How Components Capture Pixels' Correlations}

\begin{tabular}{ccccc}
\hline Modality & Domain & $P\left(\Lambda_{s}\right)$ & $\pi_{s}$ & MRF \\
Gray & Cont. & $\times$ & $\times$ & $\times$ \\
\hline
\end{tabular}

In this experiment, we used a database (Graham and Allinson 1997) of 250 images of 18 subjects, each acquired at 25 different head poses (see some views in Figs. 2 and 4). The poses in the images were manually labeled with the estimated out-of-plane rotation angle (from 0 to $45 \mathrm{deg}$ ). In fivefold cross-validation, we trained both PCA and SCA models ( $K=2, S=7$, Gaussian palettes), and chose and tested the optimal predictor of the pose angle based on the component strengths of PCA and SCA. In the case of PCA, the predictors we considered used up to the 6 components with highest eigenvalues, and, furthermore, to allow for some illumination invariance, we considered sparse variants discarding the first, the first two, or the first three components, resulting in the total of 12 different sets of top eigen-images used for prediction (i.e., [1 2], [2 3], [3 4], [4 5], [ [ 6 6], [l 2 2 3],

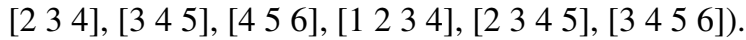

For both PCA and SCA angle prediction, the crossvalidation included linear regressor, robust linear regressor, and the nonlinear regressor.

SCA components outperformed the PCA projection as the input to regressors in this test, as the average test error for the optimal PCA-based regressor was $9.2 \mathrm{deg}$, and the optimal SCA-based regressor had a test error of $7.8 \mathrm{deg}$. Moreover, the standard deviation over the folds was twice lower for SCA, and the difference between methods was statistically significant.

As illustrated in Fig. 4, SCA does not use the single degree of freedom in the subspace to model the illumination differences, since it is palette-invariant. Rather, stels capture facial parts of relatively uniform color (therefore, uniform surface normal quite often), and the variations in $y_{1}$ captures the changes of these parts as they undergo significant geometric changes (see also Fig. 2c). Instead, the PCA model captures small geometric transformations as well as large illumination changes, but fails to capture significant structural changes, and no single PCA component captures the majority of the angle variations. In fact, the strength of the most predictive component yielded a prediction error of $13.0 \mathrm{deg}$ vs $7.8 \mathrm{deg}$ of SCA, and instead the angle has to be inferred from multiple components, and this results still lies behind SCA's single component inference.

\subsubsection{A Comparison Between SCA's Components and the Centers of a Mixture Model}

\begin{tabular}{ccccc}
\hline Modality & Domain & $P\left(\Lambda_{s}\right)$ & $\pi_{s}$ & MRF \\
Gray & Cont. & $\times$ & $\times$ & $\times$ \\
\hline
\end{tabular}

To illustrate that SCA captures the variability in the data in a substantially different way than a mixture of PIMs (MPIM, Jojic and Caspi 2004) of the same complexity, we considered the Daimler dataset (Munder and Gavrila 2006). The full dataset is composed by 10200 gray levels images at a resolution of $18 \times 36$ pixels. It is used for pedestrian classification. Pedestrian images were obtained from manually labeling and extracting the rectangular positions of pedestrians in video images. The dataset is challenging because the negative samples (non-pedestrians) present an elongated structure, easily confusable with a pedestrian. For our experiments we randomly chose a subset of 1000 positive and 
1000 negative images using half of them to train the models, the rest for testing. We repeated this process 5 times.

We learned a SCA and MPIM models with $S=5$, which broke image patches into five stels, and $K \in 1,2,3$ which represents the number of centroids for MPIM, and the number of components for SCA.

An increase in $K$ improved performances for both: MPIM, that assumes that each image is modeled by only one of the $K$ components, and the SCA model, which mixes the components differently for each image placing it in a simplex of dimension $K$, to achieve a better and spatially nonuniform blend of components.

However, SCA outperforms MPIM model, with statistical significance in several aspect. First, at the maximum complexity ( $K=3$ ), we obtained an area under the curve, AUC, of 0.9334 vs 0.9122 in favor of SCA. Second, MPIM has not improved significantly from $K=2$ to $K=3$, AUC was 0.9110 and 0.9122 , respectively, whereas SCA has reached an AUC of 0.9221 at $K=2$ and of 0.9331 at $K=3$. This further illustrates the benefits of spatial mixing of components in SCA-expressive power of the model grows much faster with $K$, despite the same number of model parameters. $^{3}$

Finally we want to highlight how our way of mixing different components is very different from linear mixing, as the latter reduces to a mixture model. Linear combination of probability maps is just a mixture. But it is too rigid a model, and so the learned probability maps, in order to accommodate for the variation in the data, end up too uncertain (blurred). A better mixing model allows more freedom in mixing, which is now spatially varying, but this then results in more certain components. Overall, the latter is better as seen in the experiments.

\subsection{Segmentation}

Using SCA for segmentation, allows one to extract an object only assuming its presence in the image. We show how an increase of the number of components $K$ yields to better segmentation results.

\subsubsection{Foreground Extraction from Videos}

\begin{tabular}{ccccc}
\hline Modality & Domain & $P\left(\Lambda_{s}\right)$ & $\pi_{s}$ & MRF \\
Color & Cont. & $\checkmark$ & $\times$ & $\checkmark$ \\
Opt.Flow & Disc. & $\checkmark$ & $\times$ & $\checkmark$ \\
\hline
\end{tabular}

To show at which extent, SCA can deal with rigid transformation we extracted the foreground from the same video

\footnotetext{
${ }^{3} \mathrm{SCA}$ has an extra layer of variables, a, but these are integrated out.
}

sequence used to test the hierarchical model selection strategy of Jojic et al. (2006). This sequence is composed by 220 frames and contains significant illumination changes, background clutter, various and confusing foreground and background motion, as well as dramatic changes in the size and pose of the foreground object (Fig. 5a). To analyze the frames of this video using our model, we used two modalities for the local pixel measurements: real valued color (mixture of Gaussians palette) and optical flow (discrete observation) for each pixel. The model complexity was set to $S=3$, $K=3, C=3$ turning off the priors on $s$. The comparison is based on the manual segmentation into foreground $(F G)$ and background $(B G)$ of one frame out of 10 . The inferred parameters concerning real valued color for the Larry video are shown in Fig. 5 where $\beta_{s, c}$ is the Dirichlet prior on the mixing coefficients $\pi_{s, c}^{t}, \mu_{0_{s, c}}$ and $\phi_{0_{s, c}}$ is the Gaussian prior on the palette means $\mu_{s, t}^{t}$, and $\Gamma$ is the inverse gamma prior on the palette variance $\phi_{s, t}^{t}$. Note how in this case the prior avoids the full color invariance helping the segmentation as adjacent frames have similar color. The parsing of our model agrees with the ground truth in $95 \%$ of pixels, result comparable with that achieved by the algorithm in Jojic et al. (2006), which is based on a much more complex hierarchical model with multiple components specialized for video processing. We also show the segmentation that SCA achieves if temporal correlations among components $y_{k}$ are modeled using a simple Brownian motion model (see Fig. 5, rows D at right). In this case, our model achieves an accuracy of $96 \%$, outperforming (Jojic et al. 2006).

To further test the SCA ability to deal with misalignments of the object in video frames, in order to allow to track it, exploiting the ability of the transformed PIM model (TPIM) (Jojic and Caspi 2004) to do the same.

The latter approach is much more computationally intensive, as it requires a search over many possible image transformations (Frey and Jojic 2003). Even when this search is sped up in case of image translations by reducing many operations to efficiently compute convolutions, the computational burden of TPIM is significantly higher than that of SCA, whose computational cost grows only linearly with the number of components $K$, and typically only a handful of components is used to manage a wide variety of geometric changes in stels. In addition to Larry video, the two approaches were compared on other 2 video sequences: one, MSRiu, has been used in Yin et al. (2007), the other one, anaivana, is available upon request (see some frames in Fig. 5). MSRiu video is characterized by a relevant FG (object) translation; instead, in anaivana, there is significant change of the FG scale.

In all cases, the FG segmentation using SCA is at least as good as the one achieved by a more expensive search over alignments performed by TPIM. For TPIM, we considered 
Larry Video Parameters $-\mathrm{S}=3, \mathrm{~K}=3, \mathrm{C}=3$

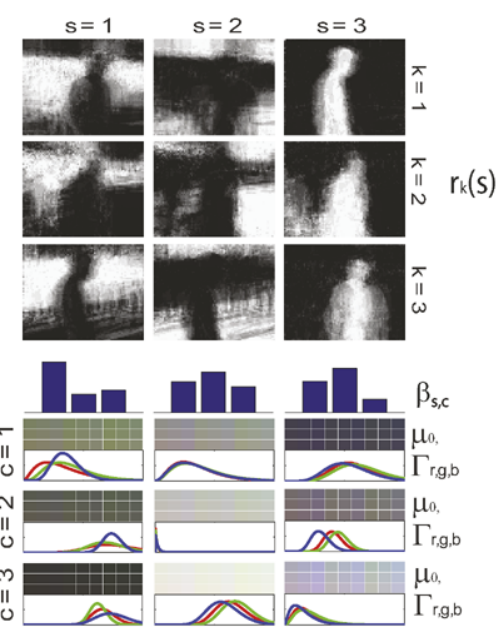

Video Segmentation Results

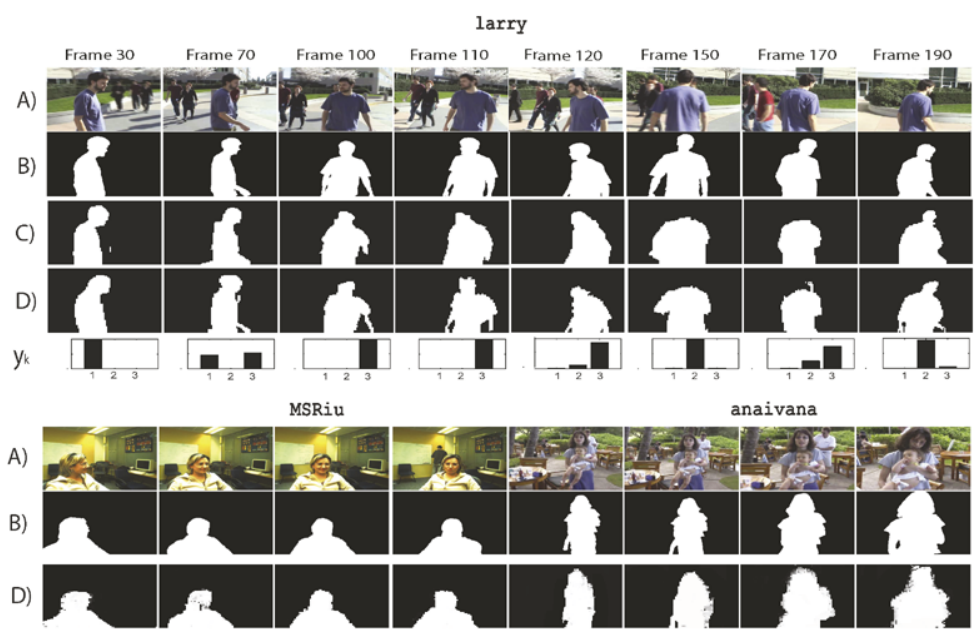

Fig. 5 Video segmentation using SCA. Left: the learned components and the color priors. Right: video segmentation results for three videos larry, MSRiu, and anaivana. For the larry video, the rows represent: (A) Frames, (B) Ground truth masks, (C) (Jojic et al. 2006) results, (D) SCA with temporal smoothing of $y_{k}$

the Weizmann horses dataset (327 horses, Borenstein and Ullman 2004). This is a standard benchmark to evaluate an algorithm segmentation accuracy (Alexe et al. 2010; Cao and Li 2007; Rother et al. 2004). LOCUS (Winn and Jojic 2005) reports only results on the Weizmann horses.

To compute the accuracy, we segmented one class at time: we learned a model using the training set and we inferred the posterior distribution $q(s)$ for the test set.

Given the ground truth data, there is one out of two possible labels for each pixel $l_{i j} \in\{0,1\}$, where 0 refers to background $(B G)$ and 1 refers to foreground $(F G)$. After the learning phase, we have also $S$ labels for each pixel based on the model $s_{i j} \in\{1, \ldots, S\}$. These labels are probabilistic, so we have $q\left(s_{i j}=s\right)$ rather than just a discrete $s_{i j}$. To create a correspondence between $s$ and $l$, we need a mapping $s \rightarrow l$ to evaluate the segmentation. Since a small value for $S$ is used by the algorithm $(2,3$ or 4$)$, it is reasonable to consider as result the best mapping. ${ }^{4}$

Figure 6 shows the learned model for the Weizmann horses. Note how components capture the salient poses; many other poses are obtained by blending them. Segmentation accuracies are reported in Table 2; they are measured by the percentage of pixels in agreement with the ground truth segmentation.

On Caltech 101, SCA outperforms (Alexe et al. 2010; Cao and Li 2007; Rother et al. 2004) setting the new state of the art on segmentation using pixels. On Weizmann horses SCA does not reach the LOCUS performance $(93.2 \%)$.

\footnotetext{
${ }^{4}$ This operation is performed only once, for all the images and the same happens in LOCUS for example, were a prior one cannot know which one of the 2 segments represent the foreground.
}

To test the ability of SCA to extract objects from images we considered Caltech 101 annotations (101 Classes, more than 30 exemplars per class, Fei-Fei et al. 2006) and 
Fig. 6 Image segmentation using SCA. We show the parameters for the Weizmann horses on the left, and some segmentation results on the right. Note how the components capture the characteristic poses of the object class

Table 2 Image segmentation results
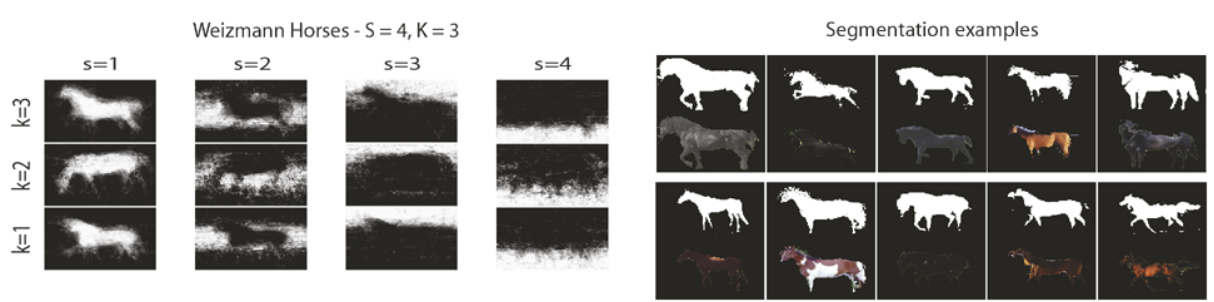

\begin{tabular}{llllll}
\hline $\begin{array}{l}\text { SCA } \\
K=1\end{array}$ & SCA & LOCUS & $\begin{array}{l}\text { ClassCut } \\
\text { (Alexe et al. } \\
\text { 2005) }\end{array}$ & $\begin{array}{l}\text { GrabCut } \\
\text { (Rother et al. } \\
\text { 2004) }\end{array}$ & $\begin{array}{l}\text { SCTM } \\
\text { (Cao and Li } \\
\text { 2007) }\end{array}$ \\
\hline $\begin{array}{l}\text { Caltech segmentation } \\
84.71 \%\end{array}$ & $88.35 \%$ & n.a. & $83.60 \%$ & $81.50 \%$ & $67.00 \%$ \\
$\begin{array}{l}\text { Weizmann horses } \\
86.54 \%\end{array}$ & $90.21 \%$ & $93.10 \%$ & $86.20 \%$ & $85.80 \%$ & $81.80 \%$ \\
\hline
\end{tabular}

Nevertheless LOCUS (1) cannot recognize horses facing opposite directions (in Winn and Jojic (2005) images have been preprocessed to solve this issue). SCA, like (Cao and Li 2007), does not need to make such assumption, it only needs an appropriate number of components $K>1$, so that the various poses of the objects can be captured by one component $r_{k}(s)$. Moreover (2) LOCUS also uses the edges and (3) it deals explicitly with deformations and transformation and it is characterized by several submodules specialized for image segmentation making it very complex. Please also note how SCA's component could also be employed by LOCUS probably further increasing its effectiveness.

The last consideration is on the components. As Table 1 shows, increasing the number of components $K$ also increases the accuracy as the model becomes more expressive. The difference is statistically significant at $p \leq 0.05$.

\subsection{Object Recognition}

\subsubsection{Generative Classification and "S-Bags of Words"}

\begin{tabular}{ccccc}
\hline Modality & Domain & $P\left(\Lambda_{s}\right)$ & $\pi_{s}$ & MRF \\
Color & Cont. & $\checkmark$ & $\times$ & $\times$ \\
SIFT & Disc. & $\checkmark$ & $\times$ & $\times$ \\
\hline
\end{tabular}

To test the performance of our model, we have trained a variety of SCA models $(S \in\{1,3,5\}, K \in\{1,2\})$, for the 2117 images of the Caltech 28 dataset.

Caltech 28 was introduced in Cao and Li (2007), and it is composed by 28 object classes, selected among the subset of Caltech 101 categories that contain more than $60 \mathrm{im}$ ages per class. These categories contain objects with thin regions (e.g., flamingo, lotus), peripheral structures (e.g., cup), not well centered objects (e.g., leopards, dalmatians, Joshua trees) and, most important, it does not contain classes characterized by background artefact that makes them easily identifiable.

For each class, we randomly select 30 images for training and 30 images for testing. SIFT features were extracted from $15 \times 15$ pixel windows computed over a grid spaced 5 pixels. At the end, these features were mapped to 300 codewords.

We learnt a model for each class and we assigned a sample to the class that provides the lowest free energy. The process is illustrated in Fig. 7 were for two classes (trees and faces) we show, the inferred parameters, the prior and the posterior of a negative and a positive sample.

Results are reported in Table 3: once again it is evident how the increasing the components help recognition. The number of stels does not affect the accuracy, being it more related to the particular object class. Cao and $\mathrm{Li}$ (2007) seems to outperform SCA's generative classification, but it requires the foreground masks for each training object.

As second test we only used discretized SIFT features as observation $\left(z_{\text {sift }}\right.$, in Fig. 7). As visible in Table 3 (Row $\mathrm{SCA}^{\text {sift }}$ ), the accuracy raises of more than $10 \%$. Moreover the model now is more efficient as the images are smaller (SIFT are computed on a grid, every 5 pixels). We repeated the test only using color but the accuracy got worse.

As further test, we illustrate the value of spatial parsing of the categories into stels. Therefore, for support vector machines without any spatial structure, we show the results obtainable by utilizing the inferred stel segmentation to relearn separate models in meaningful image parts.

We performed 1-vs-All classification using support vector machines with linear kernel (Lin.) and histogram intersection kernel (HI), concatenating all the entries of the SIFT palettes and using them as image signature: 
Fig. 7 Object recognition illustration. Top: Model parameters for the face and the tree categories $(S=4, K=3$ ) are compared with the BoW model $(S=1)$. The learned Dirichlet priors for color, and $\operatorname{SIFT}\left(\alpha_{s i f t}\right)$ are illustrated with bars whose height is proportional to the strength $\alpha_{j}$ of the different words $\zeta_{j}$. The rich histogram priors for the standard BoW model (special case of our model with $s=1$ ) are broken into tighter priors for appropriately estimated stels $s=1, \ldots, 5$. Bottom: Inferred hidden variables under the learned models for two images
Parameters

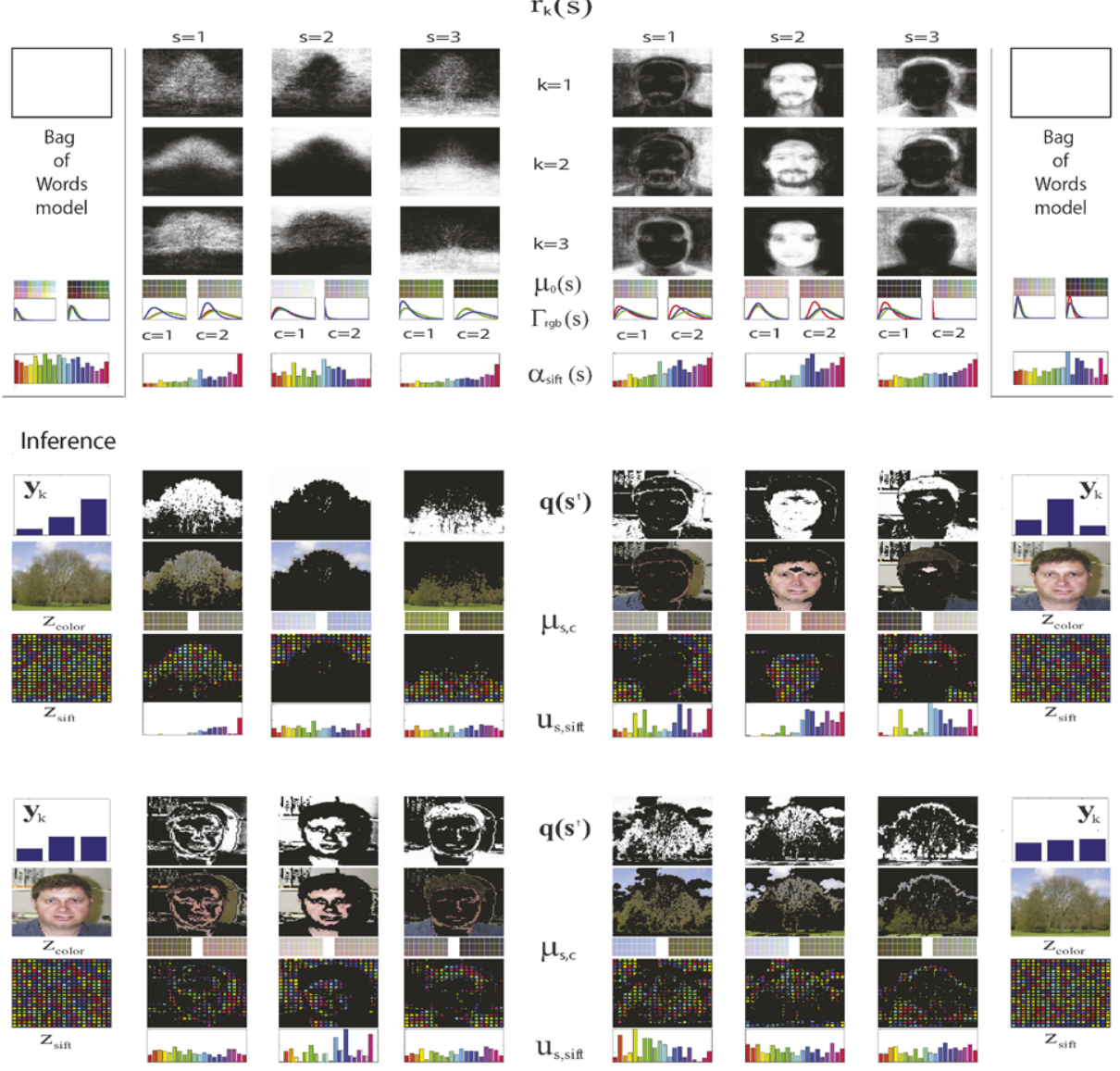

Table 3 Object recognition results. We report SCA generative classification accuracies varying $S$ and $K$. Note that for $S=1$ we have a bag-of-word generative classifier. SCA ${ }^{\text {sift }}$ only uses discretized sift as observations

\begin{tabular}{llll}
\hline & $S=1$ & $S=3$ & $S=5$ \\
\hline SCA $K=1$ & $12.31 \%$ (Dance et al. 2004) & $49.30 \%$ & $45.54 \%$ \\
SCA $K=2$ & n.a. & $54.34 \%$ & $61.39 \%$ \\
SCA $^{\text {sift }} K=2$ & n.a. & $70.12 \%$ & $73.23 \%$ \\
SPK (Lazebnik et al. 2006) & $65.40 \%$ & n.a. & n.a. \\
SCTM (Cao and Li 2007) & $69.30 \%$ & n.a. & n.a. \\
LDA (Fei-Fei and Perona 2005) & $12.32 \%$ (Fei-Fei and Perona 2005) & $34.98 \%$ & $32.21 \%$ \\
S-Bag of Words, HI Kernel & $56.40 \%$ (Dance et al. 2004) & $65.12 \%$ & $68.21 \%$ \\
S-Bag of Words, Lin. Kernel & $51.21 \%$ (Dance et al. 2004) & $61.07 \%$ & $64.64 \%$ \\
\hline
\end{tabular}

$x^{t} \rightarrow\left[u_{s=1, s i f t}^{t, c}, u_{s=2, s i f t}^{t, c}, \ldots, u_{s=S, s i f t}^{t, c}\right]$

\subsubsection{The Stel Kernel}

\begin{tabular}{ccccc}
\hline Modality & Domain & $P\left(\Lambda_{S}\right)$ & $\pi_{s}$ & MRF \\
Color & Disc. & $\times$ & $\checkmark$ & $\times$ \\
\hline
\end{tabular}

When we tried to classify all the 101 classes of Caltech (Fei-Fei et al. 2007) using the "S-Bags of words" approach, the accuracy dropped to $25.93 \%$ and $33.52 \%$ respectively for the linear and histogram intersection kernels. 
a)

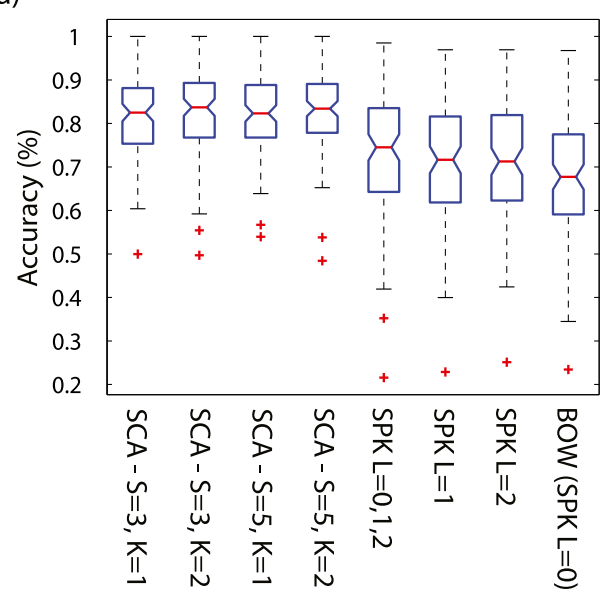

b)



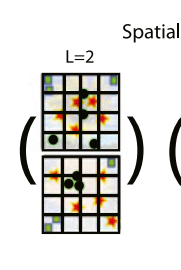

Spatial Pyramid Matching

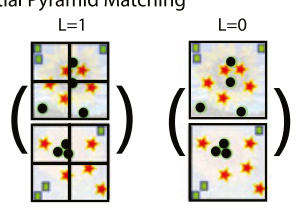

Hierarchical Stel Matching

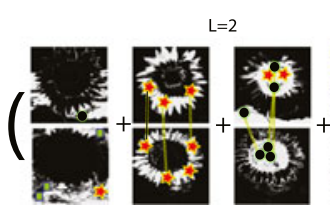

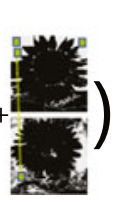

Stel Matching

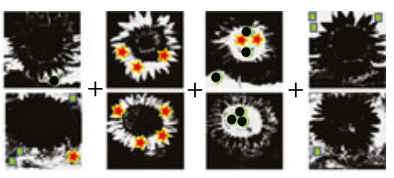

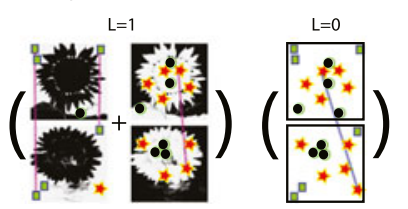

Fig. 8 On the left comparisons between the one-vs-all tests on Caltech 101. SCA outperforms with a large margin the competitors and an increase of the components yield to an increase in accuracy. On the right the four feature matching schemes considered to compute the kernels. We are considering the histogram intersection therefore we are counting the number of feature matches in each segment. Two features match if they have the same index (symbol in the figure)

the Anova tests; the central red mark is the median, the edges of the box are the 25 th and 75 th percentiles, the whiskers extend to the most extreme data points not considered outliers, and outliers are plotted individually with a red cross. As visible, in separate one-vs-all tests, "S-Bags of words" outperforms with a very large margin the other methods. These differences are statistically significant ( $p$-values lower than $0.05)$.

The problem in combining the classifiers decisions is probably due to the fact that each support vector machine, is learned with a different kernel: this results in scaling problems. This not happens for (Lazebnik et al. 2006; Dance et al. 2004) where the partition is fixed, the kernel is computed just once and only the labels are changed to learn the SVMs.

Fortunately we can improve the results employing the same technique of the Hierarchical Stel Kernel (HSK, Perina et al. 2010). Here the authors, firstly presented the hierarchical stel model (hSM), a relative of PIM based on a linked hierarchy of stel segmentations. Each level of the stel-hierarchy is characterized by a different number of stels. Exploiting those hierarchical segmentations, they defined a kernel $K_{c}$ as the weighted sum of the histogram intersections in each stel (see Fig. 8). As in Lazebnik et al. (2006) they added the level 0 (Bag of Words), and they weighted differently each level to reward more the similarities in the higher, finer ones. Being based on stel models, each class induces a kernel; the hierarchical stel kernel (HSK) is defined as the sum of all the kernels induced by the classes.

The most surprising finding of Perina et al. (2010) is that this sum of kernels yields to a pixel-wise weighting scheme. A feature match between the $i$-th pixel in one image and $j$-th

\footnotetext{
${ }^{5}$ We used 15 training images.
} 
Table 4 Stel Kernel Results (15 training images)

\begin{tabular}{llll}
\hline & $S=1$ & $S=3$ & $S=5$ \\
\hline SCA $K=1$ & $33.02 \%$ (Dance et al. 2004) & $53.99 \%$ & $54.05 \%$ \\
SCA $K=2$ & n.a. & $54.12 \%$ & $55.03 \%$ \\
\hline SPK (Lazebnik et al. 2006) & $53.41 \%$ & n.a. & n.a. \\
HSK (Perina et al. 2010) & & $58.92 \%$ & \\
\hline
\end{tabular}

Table 5 Caltech 101 Dataset: best multikernel results based on (Lazebnik et al. 2006)

\begin{tabular}{llll}
\hline Method & No. features & No. kernels & Best accuracy \\
\hline Gehler and Nowozin (2009) & 8 & 39 & $70.4 \%$ \\
Bosch et al. (2007) & 4 & 4 & $70.4 \%$ \\
Vedaldi et al. (2009) & 6 & 7 & $71.1 \%$ \\
Yang et al. (2009) & 5 & 10 & $73.2 \%$ \\
\hline
\end{tabular}

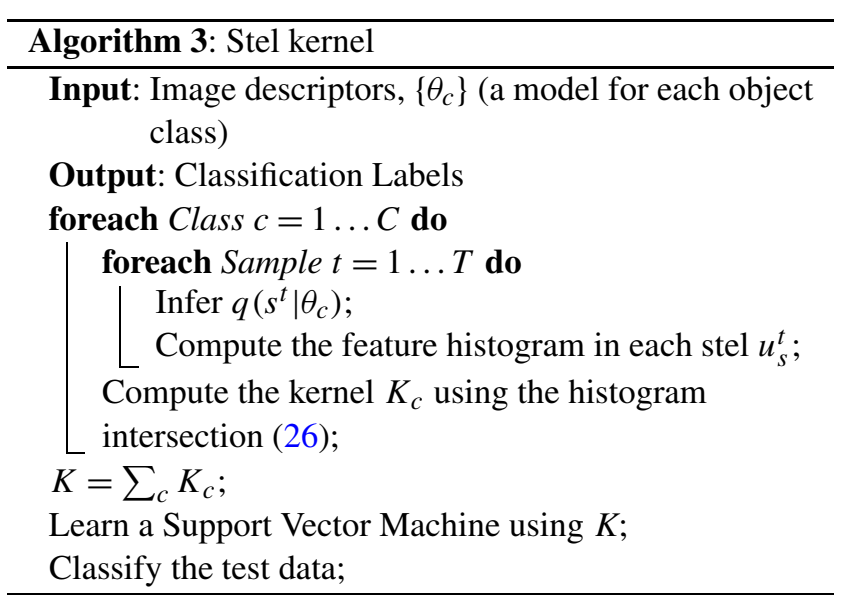

pixel in the other, is weighted by how many times the two locations share the same stel across the hierarchy of classes.

As Perina et al. (2010) we have that each class induces a kernel $K_{c}$ defined as

$K_{c}(A, B)=\sum_{s} \sum_{k} \min \left(u_{s}^{A}(k), u_{s}^{B}(k)\right)$.

The final kernel, named the "Stel Kernel" (SK) is defined as the sum of the kernels.

$K^{S K}=\sum_{c} K_{c}(A, B)$.

The classification procedure is also shown in Algorithm 3.

It is straightforward to understand how all the properties of the HSK are valid also for the present case.

The differences between HSK and SK are that SK do not consider the level 0 (bag of words), and it presents a single segmentation for each class. On the other hand, HSK does not capture correlations and therefore it is computed from poorer segmentations.

Recognition accuracies for the full Caltech dataset are shown in Table 4; for the sake of comparison, we re-run the algorithm of Lazebnik et al. (2006) with our features. ${ }^{6}$ We randomly select 30 images from each category: 15 of them are used for training and the rest are used for testing. We repeated this process 5 times and we averaged the results.

It is evident that each partition improves over the bag of features by over $20 \%$. Surprisingly also SVMs benefit from the components showing how a better segmentation helps recognition. As noted in Perina et al. (2010) for Caltech 28, the difference between SPK and SK is not statistically significant.

The algorithms which produces the best accuracies on Caltech dataset, use multiple feature and/or multikernel approaches, e.g., Bosch et al. (2007), Gehler and Nowozin (2009), Vedaldi et al. (2009), Yang et al. (2009). In Table 5 we report some statistic. All of them use the Spatial Pyramid Kernel feature organization Lazebnik et al. (2006); Table 4 demonstrates that kernels based on stel partitions outperform Lazebnik et al. (2006) with a large margin therefore it would be interesting using the stel kernel in conjunction with such methods.

\subsection{3 $q(s)$ as Feature}

\begin{tabular}{ccccc}
\hline Modality & Domain & $P\left(\Lambda_{s}\right)$ & $\pi_{s}$ & MRF \\
Color & Cont. & $\times$ & $\times$ & $\times$ \\
\hline
\end{tabular}

To investigate further the capability of SCA to identify discriminant features, we considered Caltech 101, for which the best features, used discriminatively, provide classification rates of 40-59\% as shown in Table 6. We compare our model with several others on this dataset. In Berg and

${ }^{6}$ The result reported in Lazebnik et al. (2006) (56.40\%) is slightly better than our result $(53.41 \%)$. 
Table 6 Feature comparison on Caltech 101 dataset. We used 30 training samples

\begin{tabular}{|c|c|c|c|c|c|c|c|}
\hline $\begin{array}{l}\text { Shape } \\
\text { GB 1 } \\
\text { (Berg and } \\
\text { Malik 2001) }\end{array}$ & $\begin{array}{l}\text { Shape } \\
\text { GB 2 } \\
\text { (Berg and } \\
\text { Malik 2001) }\end{array}$ & $\begin{array}{l}\text { Self } \\
\text { Sim. } \\
\text { (Shechtman and } \\
\text { Irani 2007) }\end{array}$ & $\begin{array}{l}\text { Shp } \\
180 \\
\text { (Zhang et al. } \\
\text { 2006) }\end{array}$ & $\begin{array}{l}\text { Shp } \\
360 \\
\text { (Zhang et al. } \\
\text { 2006) }\end{array}$ & $\begin{array}{l}\text { Sift } \\
\text { Col. } \\
\text { (Bosch et al. } \\
2006 \text { ) }\end{array}$ & $\begin{array}{l}\text { Sift } \\
\text { Gray } \\
\text { (Lowe 1999) }\end{array}$ & $\begin{array}{l}q(s) \\
\text { SCA }\end{array}$ \\
\hline $57 \%$ & $59 \%$ & $55 \%$ & $48 \%$ & $50 \%$ & $40 \%$ & $52 \%$ & $50.60 \%$ \\
\hline
\end{tabular}

Malik (2001), GB features correspond to geometric blur which captures some of the spatial configurations in the feature distributions, and App. Color and Gray are SIFT features calculated from color and gray-level images utilized in Bosch et al. (2006), Lowe (1999), and Self Similarity is introduced in Shechtman and Irani (2007). The rest of the features capture gradient orientations, so mostly representing local shape features (Zhang et al. 2006).

Here we only used color as a local measurement, but we performed classification using only the inferred stel segmentation, $q\left(s^{t}\right)$, without parts of the likelihood that have to do with matching of image measurements to those expected for the category. This corresponds to dropping out the first two terms from the free energy in (7), which deal with evaluating the uniformity of the observed features $z_{\mathbf{i}}$ and their agreement with the prior over the entire class defined by $\Lambda$. Therefore, the only terms kept are the last two terms concerned with the KL distance between the prior $r_{k}(s)$ and the inferred stel tessellation for the image $q\left(s^{t}\right)$. Such classification yields accuracy of $24.7 \%(18.9 \%$ at $K=1)$ against the $17.3 \%$ obtained by Fei-Fei et al. (2007), which represents the only reported result obtained by a generative model.

However, the discriminative use of the inferred stel, through SVM classification using only inferred stels as features resulted in classification accuracy of $50.62 \%$, making the global shape features defined by stel segmentation of comparable quality to the top features used in object classification. This is rather encouraging as these features capture rather different aspects of the images.

It is also important to note here that for an already trained SCA model, inference of stels $q\left(s^{t}\right)$ for any new given image consists of only a 4-5 iterations of (8)-(12), as the SCA components $r_{k}$, and palette priors are linked to the entire category, not to a single image. Thus, inference for a single image is linear in the number of pixels, and is in fact more computationally efficient than the computations involved in methods that require a large number of filter banks or SIFT extraction. SCA does not require it when the image measurement considered is just color.

\section{Conclusions}

In this paper, we have proposed a novel model able to summarize a class of object (1) segmenting images in meaningful parts (stels), (2) capturing the correlations of the spatial structure, (3) identifying interesting co-occurrences of local measurements among the images of the same class.

Instead of relying on consistency of image features across images from the same class, the model mines self similarity patterns within individual images, which helps in the inference of a consistent segmentation of images into structural elements (stels), shared across the entire class, even when the images differ dramatically in local colors and features.

Significant variations in stels can be tolerated by a subspace modeling framework, Stel Component Analysis (SCA), which captures correlated changes in image structure and thus avoids over-generalization whose the PIM model (a SCA ancestor) is prone when faced with significant structural variations. The model can be inferred from the data in only with a bounding box annotation (eventually coarse), so affording significant advantages to this image representation in a variety of computer vision tasks, some of which have been illustrated above.

SCA demonstrated good performances in modeling object classes thanks to its capability to organize and select the related features, also coping with severe different aspects of the same object, in increasing the performances of discriminative methods when applied to SCA outcomes, and in segmenting images and video sequence of a certain complexity. In all these applications, it has been shown how the components helped to improve the accuracy.

Open Access This article is distributed under the terms of the Creative Commons Attribution License which permits any use, distribution, and reproduction in any medium, provided the original author(s) and the source are credited.

\section{References}

Alexe, B., Deselaers, T., \& Ferrari, V. (2010). Classcut for unsupervised class segmentation. In Proceedings of European conference on computer vision (ECCV) (pp. 380-393).

Alexe, B., Deselaers, T., \& Ferrari, V. (2010). What is an object. In Proceedings of IEEE computer society conference on computer vision and pattern recognition (pp. 73-80).

Bagon, S., Brostovski, O., Galun, M., \& Irani, M. (2010). Detecting and sketching the common. In Proceedings of IEEE computer society conference on computer vision and pattern recognition (CVPR) (pp. 33-40).

Bay, H., Tuytelaars, T., \& Gool, L. V. (2006). Surf: speeded up robust features. In Proceedings of European conference on computer vision (ECCV) (pp. 404-417). 
Berg, A. C., \& Malik, J. (2001). Geometric blur for template matching. In Proceedings of IEEE computer society conference on computer vision and pattern recognition (CVPR) (pp. 607-614).

Blei, D., Ng, A., \& Jordan, M. (2003). Latent Dirichlet allocation. Journal of Machine Learning Research, 3, 993-1022.

Boiman, O., Shechtman, E., \& Irani, M. (2008). In defense of nearestneighbor based image classification. In Proceedings of IEEE computer society conference on computer vision and pattern recognition (CVPR) (pp. 1-8).

Borenstein, E., \& Ullman, S. (2004). Learning to segment. In Proceedings of European conference on computer vision (ECCV) (pp. 315-328).

Bosch, A., Zisserman, A., \& Munoz, X. (2006). Scene classification via plsa. In Proceedings of European conference on computer vision (ECCV) (pp. 517-530).

Bosch, A., Zisserman, A., \& Munoz, X. (2007). Image classification using random forests and ferns. In Proceedings of international conference on computer vision (ICCV) (pp. 1-8).

Buntine, W. (2002). Variational extensions to em and multinomial pca. In Proceedings of European conference on machine learning (ECML) (pp. 23-34).

Cao, L., \& Li, F. F. (2007). Spatially coherent latent topic model for concurrent segmentation and classification of objects and scenes. In Proceedings of international conference on computer vision (ICCV) (pp. 1-8).

Cheung, V., Jojic, N., \& Samaras, D. (2007). Capturing long-range correlations with patch models. In Proceedings of IEEE computer society conference on computer vision and pattern recognition $(C V P R)$ (pp. 1-8).

Chu, X., Yan, S., Li, L., Chan, K. L., \& Huang, T. (2010). Spatialized epitome and its applications. In Proceedings of IEEE computer society conference on computer vision and pattern recognition (CVPR) (pp. 311-318).

Collins, M., Dasgupta, S., \& Schapire, R. (2001). A generalization of principal component analysis to the exponential family. In $A d-$ vances in neural information processing systems (NIPS) (pp. 617624).

Cristani, M., Perina, A., Castellani, U., \& Murino, V. (2008). Geolocated image analysis using latent representations. In Proceedings of IEEE computer society conference on computer vision and pattern recognition $(C V P R)$ (pp. 1-8).

Dance, C., Willamowski, J., Fan, L., Bray, C., \& Csurka, G. (2004). Visual categorization with bags of keypoints. In Workshop on statistical learning in computer vision (in conjunction with European conference on computer vision) (pp. 1-12).

Deng, L., \& O'Shaughnessy, D. (2003). Speech processing: a dynamic and optimization-oriented approach. New York: Dekker.

Deselaers, T., \& Ferrari, V. (2010). Global and efficient self-similarity for object classification and detection. In Proceedings of IEEE computer society conference on computer vision and pattern recognition (CVPR) (pp. 1633-1640).

Fei-Fei, L., Fergus, R., \& Perona, P. (2006). One-shot learning of object categories. IEEE Transactions on Pattern Analysis and Machine Intelligence, 28, 594-611.

Fei-Fei, L., Fergus, R., \& Perona, P. (2007). Learning generative visual models from few training examples: an incremental bayesian approach tested on 101 object categories. Computer Vision and Image Understanding Journal.

Fei-Fei, L., \& Perona, P. (2005). A bayesian hierarchical model for learning natural scene categories. In Proceedings of IEEE computer society conference on computer vision and pattern recognition (CVPR) (pp. 524-531).

Frey, B., \& Jojic, N. (2003). Transformation-invariant clustering using the EM algorithm. IEEE Transactions on Pattern Analysis and Machine Intelligence, 25(1), 1-17.
Gehler, P., \& Nowozin, S. (2009). On feature combination for multiclass object classification. In Proceedings of international conference on computer vision (ICCV) (pp. 221-228).

Graham, D., \& Allinson, N. (1997). Characterizing virtual eigensignatures for general purpose face recognition. In Face recognition: from theory to applications (pp. 446-456).

Harris, C., \& Stephens, M. (1988). A combined corner and edge detector. In Proceedings of the Alvey vision conference (pp. 147-151).

Hofmann, T. (1999). Probabilistic latent semantic indexing. In Proceedings of the annual international ACM conference on research and development in information retrieval (SIGIR) (pp. 50-57).

Hofmann, T. (2001). Unsupervised learning by probabilistic latent semantic analysis. Machine Learning, 42(1/2), 177-196.

Jojic, N., Perina, A., Cristani, M., Murino, V., \& Frey, B. (2009). Stel component analysis: modeling spatial correlations in image class structure. In Proceedings of IEEE computer society conference on computer vision and pattern recognition (CVPR) (pp. 20442051).

Jojic, N., \& Caspi, Y. (2004). Capturing image structure with probabilistic index maps. In Proceedings of IEEE computer society conference on computer vision and pattern recognition (CVPR) (pp. 212-219).

Jojic, N., Caspi, Y., \& Reyes-Gomez, M. (2004). Probabilistic index maps for modeling natural signals. In Proceedings of conference on uncertainty in artificial intelligence (UAI) (pp. 293-300).

Jojic, N., Frey, B. J., \& Kannan, A. (2003). Epitomic analysis of appearance and shape. In Proceedings of international conference on computer vision (ICCV) (pp. 34-41).

Jojic, N., Perina, A., \& Murino, V. (2010). Structural epitome: a way to summarize one's visual experience. In Advances in neural information processing systems (pp. 1027-1035).

Jojic, N., Winn, J., \& Zitnick, L. (2006). Escaping local minima through hierarchical model selection: automatic object discovery, segmentation, and tracking in video. In Proceedings of IEEE computer society conference on computer vision and pattern recognition (CVPR) (pp. 117-124).

Lazebnik, S., Schmid, C., \& Ponce, J. (2006). Beyond bags of features: spatial pyramid matching for recognizing natural scene categories. In Proceedings of IEEE computer society conference on computer vision and pattern recognition (CVPR) (pp. 2169 2178).

Lee, D. D., \& Seung, H. S. (1999). Learning the parts of objects by non-negative matrix factorization. Nature, 401(6755), 788-791.

Leibe, B., Leonardis, A., \& Schiele, B. (2004). Combined object categorization and segmentation with an implicit shape model. In Workshop on statistical learning in computer vision (in conjunction with European conference on computer vision) (pp. 17-32).

Liu, T., Sun, J., Zheng, N.n., Tang, X., \& Shum, H.y. (2007). Learning to detect a salient object. In Proceedings of IEEE computer society conference on computer vision and pattern recognition (CVPR) (pp. 1-8).

Lowe, D. (1999). Object recognition from local scale-invariant features. In Proceedings of international conference on computer vision (ICCV) (pp. 1150-1157).

Marszałek, M., \& Schmid, C. (2006). Spatial weighting for bag-offeatures. In Proceedings of IEEE computer society conference on computer vision and pattern recognition (CVPR) (pp. 2118 2125).

Matas, J., Chum, O., Martin, U., \& Pajdla, T. (2002). Robust wide baseline stereo from maximally stable extremal regions. In Proceedings of British machine vision conference (BMVC) (pp. 384-393).

Mikolajczyk, K., \& Schmid, C. (2004). Scale \& affine invariant interest point detectors. International Journal of Computer Vision, 60(1), 63-86.

Munder, S., \& Gavrila, D. M. (2006). An experimental study on pedestrian classification. IEEE Transactions on Pattern Analysis and Machine Intelligence, 28(11), 1863-1868. 
Ni, K., Kannan, A., Criminisi, A., \& Winn, J. (2009). Epitomic location recognition. IEEE Transactions on Pattern Analysis and Machine Intelligence, 31(12), 2158-2167.

Perina, A., Cristani, M., \& Murino, V. (2010). 2lda: segmentation for recognition. In Proceedings of international conference on pattern recognition (ICPR) (pp. 995-998).

Perina, A., Cristani, M., Murino, V., \& Jojic, N. (2008). Capturing video structure with mixture of probabilistic index maps. In ECCV 2008 workshops: the 1st international workshop on machine learning for vision-based motion analysis—MLVMA'08, Marseille, France.

Perina, A., Cristani, M., \& Murino, V. (2010). Learning natural scene categories by selective multi-scale feature extraction. Image and Vision Computing, 28, 927-939.

Perina, A., \& Jojic, N. (2011). Image analysis by counting on a grid. In Proceedings of IEEE computer society conference on computer vision and pattern recognition (CVPR) (pp. 1985-1992).

Perina, A., Jojic, N., Castellani, U., Cristani, M., \& Murino, V. (2010). Object recognition with hierarchical stel models. In Proceedings of European conference on computer vision (ECCV) (pp. 15-28).

Quattoni, A., Collins, M., \& Darrell, T. (2004). Conditional random fields for object recognition. In Advances in neural information processing systems (NIPS) (pp. 1097-1104).

Rother, C., Kolmogorov, V., \& Blake, A. (2004). Grabcut: Interactive foreground extraction using iterated graph cuts. ACM Transactions on Graphics, 23, 309-314.

Savarese, S., \& Fei-Fei, L. (2007). 3d generic object categorization, localization and pose estimation. In Proceedings of international conference on computer vision (ICCV) (pp. 1-8).

Shechtman, E., \& Irani, M. (2007). Matching local self-similarities across images and videos. In Proceedings of IEEE computer society conference on computer vision and pattern recognition (CVPR) (pp. 1-8).

Shotton, J., Winn, J. M., Rother, C., \& Criminisi, A. (2006). TextonBoost: joint appearance, shape and context modeling for multi-class object recognition and segmentation. In Proceedings of European conference on computer vision (ECCV) (pp. 1-15).

Sivic, J., Russell, B., Efros, A. A., Zisserman, A., \& Freeman, B. (2005). Discovering objects and their location in images. In International conference on computer vision (ICCV 2005). (October 2005).

Su, H., Sun, M., Fei-Fei, L., \& Savarese, S. (2009). Learning a dense multi-view representation for detection, viewpoint classification and synthesis of object categories. In Proceedings of international conference on computer vision (ICCV) (pp. 213-220).
Sudderth, E. B., Torralba, A., Freeman, W. T., \& Willsky, A. S. (2005). Learning hierarchical models of scenes, objects, and parts. In Proceedings of international conference on computer vision (ICCV) (pp. 1331-1338).

Sun, M., Su, H., Savarese, S., \& Fei-Fei, L. (2009). A multi-view probabilistic model for $3 \mathrm{~d}$ object classes. In Proceedings of IEEE computer society conference on computer vision and pattern recognition (CVPR) (pp. 1247-1254).

Vedaldi, A., Gulshan, V., Varma, M., \& Zisserman, A. (2009). Multiple kernels for object detection. In Proceedings of international conference on computer vision (ICCV) (pp. 606-613).

Vogel, J., \& Schiele, B. (2007). Semantic modeling of natural scenes for content-based image retrieval. International Journal of Computer Vision, 72, 133-157.

Wallach, H. M., Mimno, D., \& McCallum, A. (2009). Rethinking lda: Why priors matter. In Advances in neural information processing systems (NIPS) (pp. 1973-1981).

Weber, M., Welling, M., \& Perona, P. (2000). Unsupervised learning of models for recognition. In Proceedings of European conference on computer vision (ECCV) (pp. 18-32).

Willamowski, J., Arregui, D., Csurka, G., Dance, C., \& Fan, L. (2004). Categorizing nine visual classes using local appearance descriptors. In Workshop on learning for adaptable visual systems (in conjunction with international conference on pattern recognition) (pp. 1-4).

Winn, J., \& Jojic, N. (2005). Locus: learning object classes with unsupervised segmentation. In Proceedings of international conference on computer vision (ICCV) (pp. 756-763).

Yang, J., Li, Y., Tian, Y., Duan, L., \& Gao, W. (2009). Group-sensitive multiple kernel learning for object categorization. In Proceedings of international conference on computer vision (ICCV) (pp. 436443).

Yang, Y., Hallman, S., Ramanan, D., \& Fowlkes, C. (2010). Layered object detection for multi-class segmentation. In Proceedings of IEEE computer society conference on computer vision and pattern recognition (CVPR) (pp. 3113-3120).

Yin, P., Criminisi, A., Winn, J. M., \& Essa, I. A. (2007). Tree-based classifiers for bilayer video segmentation. In Proceedings of IEEE computer society conference on computer vision and pattern recognition (CVPR) (pp. 1-8).

Zhang, H., Berg, A. C., Maire, M., \& Malik, J. (2006). Svm-knn: discriminative nearest neighbor classification for visual category recognition. In Proceedings of IEEE computer society conference on computer vision and pattern recognition (CVPR) (pp. 21262136). 